\title{
ETHNOMEDICINAL PLANTS USED AGAINST GASTROINTESTINAL DISORDERS IN NEPAL. A REVIEW
}

Ratna Silwal Gautam ${ }^{*}$, Sudha Joshi Shrestha², lla Shrestha ${ }^{3}$

1, 2, 3 Department of Botany, Patan Multiple Campus, Tribhuvan University, Kathmandu Nepal

Email : silwalratna@gmail.com

\begin{abstract}
The gastrointestinal disorders that occur in the gastrointestinal tract are of common prevalence in human beings. Traditionally used medicinal plants play vital role in curing the various disorders of the body. The present study was carried out to find the ethnomedicinal plants used for the treatment of gastrointestinal disorders in human beings. A total of 289 ethnomedicinal plant species belonging to 229 genera representing 101 families are documented from west Nepal to east Nepal. Local people in different districts of the country are using the ethnobotanical plants in the treatment of gastrointestinal problems such as cholera, diarrhoea, dysentery, gastritis, constipation, to kill worms and to increase appetite. On the basis of number of citation, Zanthoxylum armatum, Aegle marmelos, Berginia ciliata, Cannabis sativa, Chenopodium album, Cinnamomum tamala, Cynodon dactylon, Cyperus rotundus, Drymaria cordata, Mangifera indica, Melia azadirchta, Oxalis corniculata, Plantago major, Rubus ellipticus, Syzygium cumini, Woodfordia fruticosa, and Zingiber officinalae were found important ethnomedicinal plants used in the treatment of gastrointestional disorders. As the knowledge about the usage of the ethnomedicinal plants are eroding, proper documentation and conservation of such plants and traditional ethnomedicinal knowledge of local people is needed.
\end{abstract}

Key words: Genera; Conservation; Constipation; Treatment; documentation. 


\section{INTRODUCTION}

People residing in rural areas have a close association with the natural resources of their surrounding and possess hands-on knowledge about various uses of the plants and plant parts around them. The ethnic communities have significant knowledge on utilization of plants and plant parts and there is a long tradition of transferring this indigenous knowledge from generation to generation (Acharya and Acharya, 2009). Plants are the primary health care resource in many communities around the world (Bannerman et al., 1983). Of the 75,000 plants used in different systems of medicine, more than 20,000 species of higher plants are used in the traditional treatment practices of indigenous cultures living around the world (Ved Prakash, 1998). In Nepal, about $80 \%$ of the rural communities depend upon herbal plants as medicine for their primary primary health care (Ghimire et al., 1999). It is estimated that about 1700 species of the plants are utilized by different ethnic groups in Nepal to meet their primary healthcare needs (Rawal, 2004). Nepal is rich in both traditional knowledge as well as natural resources (Koirala et al., 2009). Documentation of ethnomedicinal use of plants has been considered a high priority to support the discovery of new drugs to benefit mankind (Ghimire and Bastakoti, 2009). Gastrointestinal disorders are the any condition or disease that occurs within the gastrointestinal tract of the body and have a high prevalence in human beings. The important gastrointestinal problems are diarrhea, dysentery, gastritis, constipation and parasites (Kasper et al., 2005). Use of ethnomedicinal plants to cure gastrointestinal disorders is still common in many ethnic communities in developing country like Nepal. The present study aimed to document the ethnomedicinal plants used in the treatment of gastrointestinal disorders in human beings, their mode of use and frequency index of those plants.

\section{Life forms of ethnomedicinal plants used in gastrointestinal disorders}

Altogether 289 species of ethnomedicinal plant species from 101 families and 229 genera are documented from Nepal (Table 1). Regarding the life forms, out of the 289 species, 24 species (9\%) are climbers, 148 species (51\%) are herbs, 44 species (15\%) are shrubs and 73 species $(25 \%)$ are trees (Figure 1; Table 1). Among them, 96\% (278 spp.) of the total recorded ethnomedicinal plants used to treat gastrointestinal disorders are angiosperms (240 spp. of dicots \& 38 spp. of monocots), $2 \%$ are gymnosperms and remaining $2 \%$ are pteridophytes (Figure 2). 


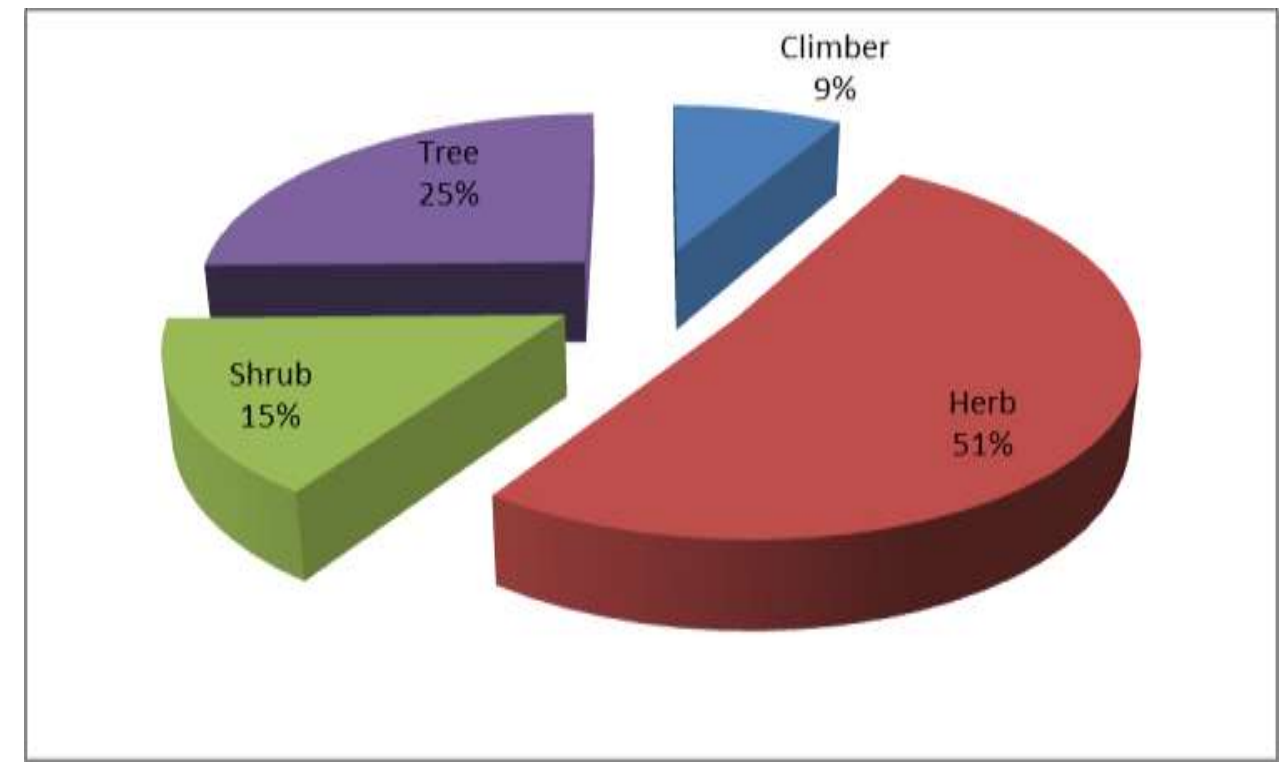

Figure 1. Life forms of ethnomedicinal plants

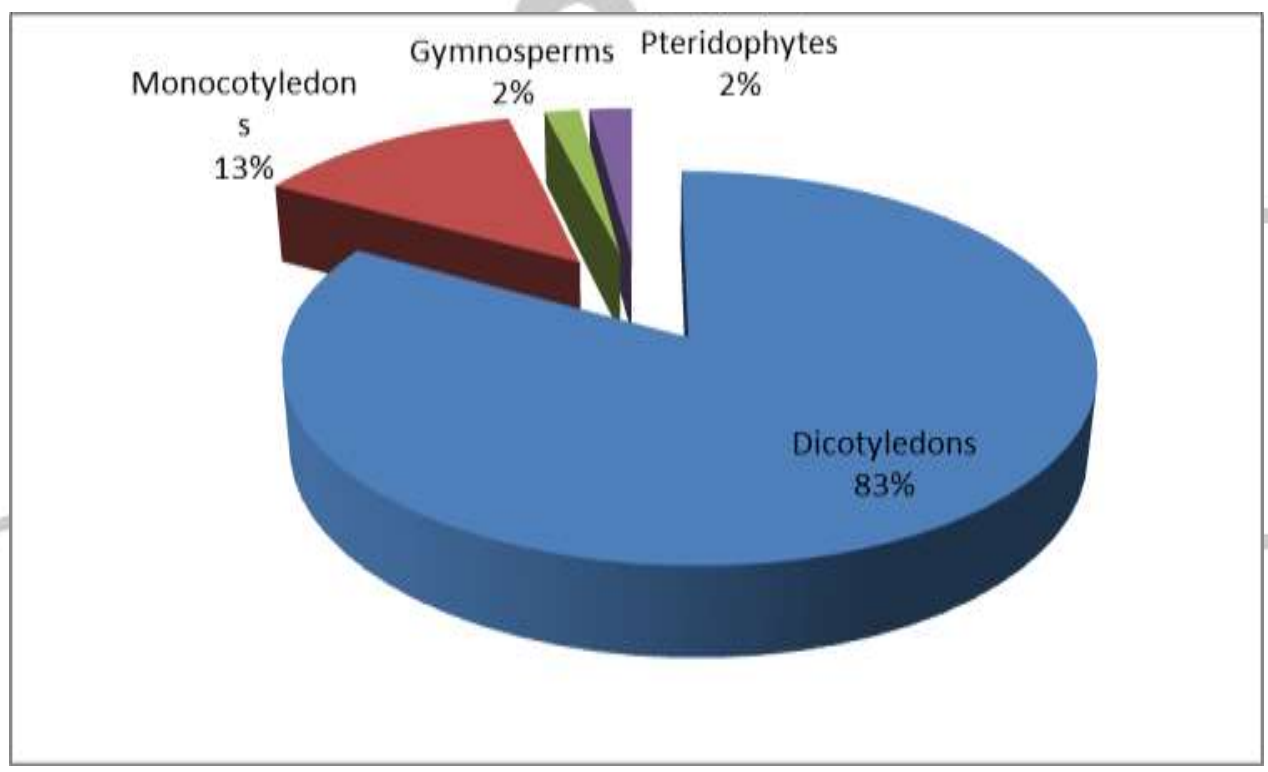

Figure 2. Types of ethnomedicinal plants

\section{Families of ethnomedicinal plants used in gastrointestinal disorders}

51 families are with more than one ethnomedicinal plants used to treat gastrointestinal disorders and remaining 49 families have single species of ethnomedicinal plant species. Fabaceae was found to have the highest number of species i.e. 20 spp. followed by Asteraceae with 15 spp., Polygonaceae, Rosaceae, and Ranunculaceae having 11 spp. For each, Zingiberaceae 9 spp., Apiaceae, Rutaceae, and Lamiaceae having 8 spp. each and so on (Figure 3). 


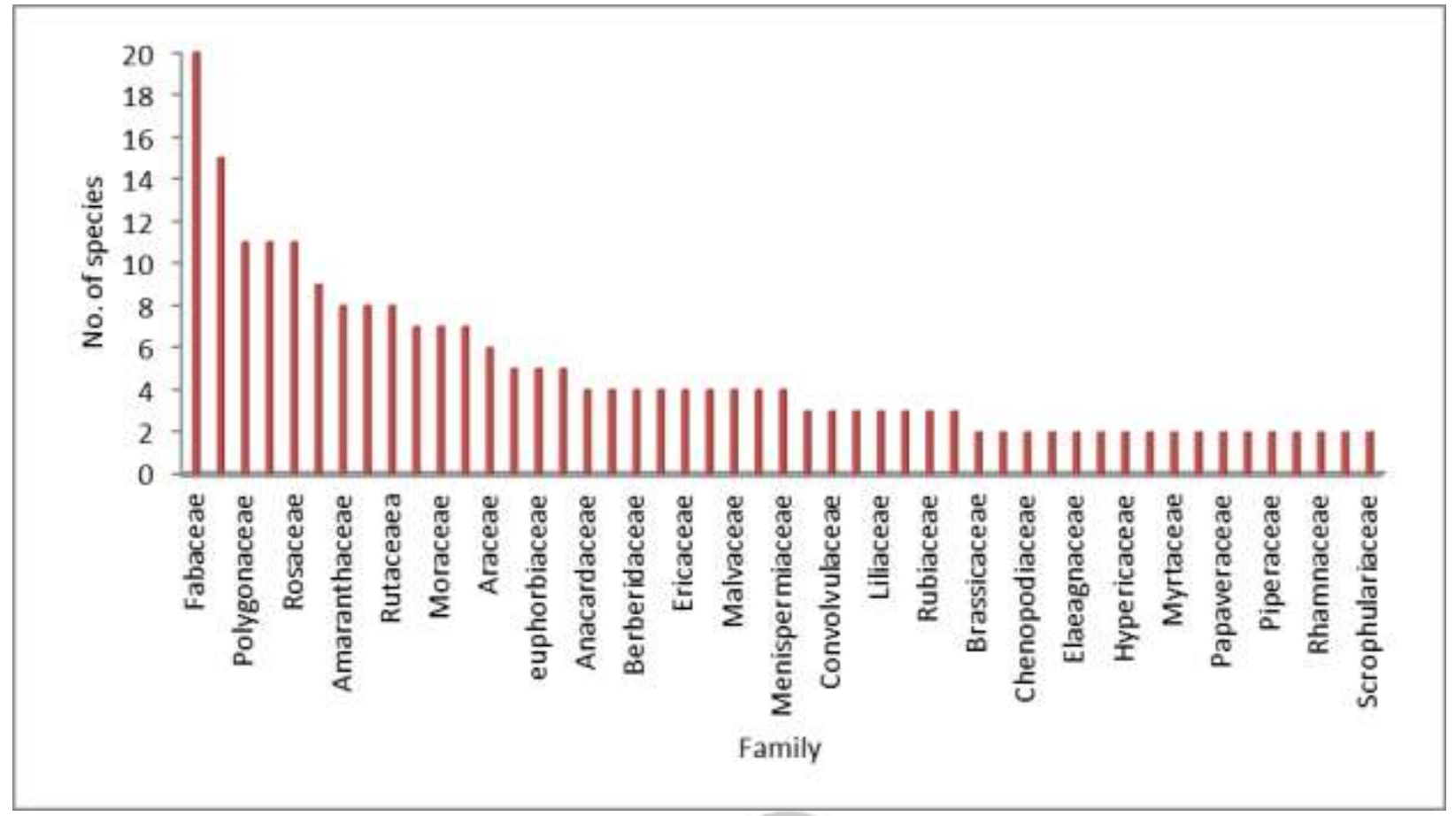

Figure 3. Families having more than one species

\section{Use category}

The study revealed that different ethnomedicinal plant parts were used in the treatment of gastrointestinal disorders. The plant parts such as fruit, leaves, shoots, bark, stem, spore, bulbils, flower, latex, resin, seed, and underground parts like root/rhizome/bulb / tuber and in some cases whole plant are used for the treatment. The most commonly used plant parts were underground parts (74 spp.), whole plant (47 spp.), fruits (43 spp.), leaves (29 spp), bark of 23 spp. and so on (Figure 4). In case of other plant species two or more than two plant parts are used for the treatment.

Among the total of 289 ethnomedicinal plant species, the sixteen plant species were identified as important on the basis of highest citations. These are Zanyhoxylum armatum, Woodfordia fruticosa, Rubus ellipticus, Plantago major, Oxalis corniculata, Drymaria cordata, Cyperus rotundus, Cinnamomum tamala, Aegle marmelos, Cannabis sativa, Chenopodium album, Cynodon dactylon, Mangifera indica, Melia azadirchta, Syzygium cumini and Zingiber officinale. 


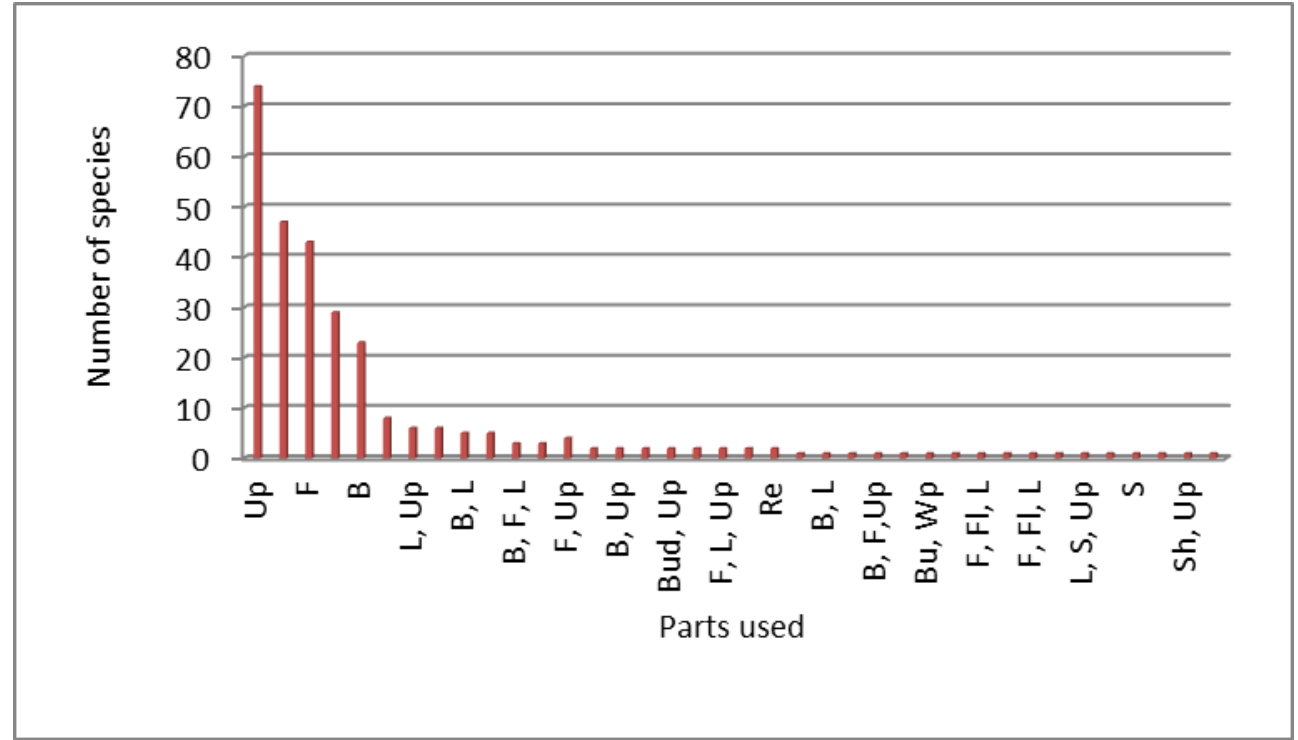

Figure 4. Parts used and number of species

Table 1. List of ethnomedicinal plant species used in the treatment of gastrointestinal disorders.

\begin{tabular}{|c|c|c|c|c|c|c|c|c|}
\hline S.no & Scientific name & Nepali name & Family & Habit & $\begin{array}{l}\text { Used } \\
\text { parts }\end{array}$ & Uses & References & $\begin{array}{l}\text { Citation } \\
\mathrm{s}\end{array}$ \\
\hline 1. & Acacia catecheu $($ L.f)Willd & Khayer & Fabaceae & $\mathrm{T}$ & $\mathrm{B}, \mathrm{S}$ & $\begin{array}{l}\text { Wood decoction } \\
\text { and bark powder is } \\
\text { given orally in } \\
\text { intestinal pain, } \\
\text { diarrhoea and } \\
\text { dysentery. }\end{array}$ & $\begin{array}{l}\text { Singh et al., } \\
\text { 2018; Dhami, } \\
2008\end{array}$ & 2 \\
\hline 2. & $\begin{array}{l}\text { Acacia nilotica (L.) Willd. Ex } \\
\text { Del. }\end{array}$ & Babool & Fabaceae & $\mathrm{T}$ & $\mathrm{B}, \mathrm{S}$ & $\begin{array}{l}\text { Stem and bark is } \\
\text { boiled and juice is } \\
\text { taken in diarrhoea } \\
\text { and dysentery. }\end{array}$ & $\begin{array}{l}\text { Singh et al., } \\
2018\end{array}$ & 1 \\
\hline 3. & Achyranthes asper $\mathrm{L}$. & & Amaranthaceae & $\mathrm{H}$ & Up & $\begin{array}{l}\text { Root juice or } \\
\text { decoction is given } \\
\text { in diarrhoea, } \\
\text { dysentery and } \\
\text { constipation. }\end{array}$ & $\begin{array}{l}\text { Dhami, 2008; } \\
\text { Bhattarai et al., } \\
2009\end{array}$ & 2 \\
\hline 4. & Acmella calva (DC.) R.K. Jansen & & Asteraceae & $\mathrm{H}$ & $\mathrm{F}$ & $\begin{array}{l}\text { Fruit is used in } \\
\text { gastritis and } \\
\text { intestinal worms. }\end{array}$ & $\begin{array}{l}\text { Adhikari et al., } \\
2019\end{array}$ & 1 \\
\hline 5. & $\begin{array}{l}\text { Acmella paniculata (Wall. Ex } \\
\text { DC.) R.K.Jansen }\end{array}$ & Bhuitimur & Asteraceae & $\mathrm{H}$ & $\mathrm{Fl}$ & $\begin{array}{l}\text { Flower juice is } \\
\text { given in stomach } \\
\text { pain. }\end{array}$ & $\begin{array}{l}\text { Singh et al., } \\
2018\end{array}$ & 1 \\
\hline 6. & Aconogonon molle (D.Don) Hara & Thotne & Polygonaceae & $\mathrm{H}$ & $\mathrm{Sh}$ & $\begin{array}{l}\text { Shoot infusion is } \\
\text { consumed to } \\
\text { relieve intestinal } \\
\text { pain. }\end{array}$ & $\begin{array}{l}\text { Rokaya et al., } \\
2010\end{array}$ & 1 \\
\hline 7. & $\begin{array}{l}\text { Aconitum bisma (Buch.-Ham.) } \\
\text { Rapaics }\end{array}$ & Bikh & Ranunculaceae & $\mathrm{H}$ & $\mathrm{Wp}$ & $\begin{array}{l}\text { Powder of dry } \\
\text { plant or fresh plant } \\
\text { paste is used in } \\
\text { diarrhoea and } \\
\text { vomitting. }\end{array}$ & $\begin{array}{l}\text { Rokaya et al., } \\
2010\end{array}$ & 1 \\
\hline 8. & Aconitum ferox Wall. Ex Ser. & Seto bikhma & Ranunculaceae & $\mathrm{H}$ & Up & $\begin{array}{l}\text { Dried root is taken } \\
\text { with boiled water } \\
\text { to treat diarrhoea } \\
\text { and stomach } \\
\text { disorders. }\end{array}$ & $\begin{array}{l}\text { Singh et al., } \\
2016\end{array}$ & 1 \\
\hline
\end{tabular}




\begin{tabular}{|c|c|c|c|c|c|c|c|c|}
\hline 9. & $\begin{array}{l}\text { Aconitum heterophyllum Wall.ex } \\
\text { Royle }\end{array}$ & Atish & Ranunculaceae & $\mathrm{H}$ & $\mathrm{Up}$ & $\begin{array}{l}\text { Root powder is } \\
\text { taken with hot } \\
\text { water to cure } \\
\text { diarrhoea and } \\
\text { dysentery. }\end{array}$ & $\begin{array}{l}\text { Rokaya et al., } \\
2010\end{array}$ & 1 \\
\hline 10. & $\begin{array}{l}\text { Aconitum laciniatum (Bruhl) } \\
\text { Stapf }\end{array}$ & Kalo bikhma & Ranunculaceae & $\mathrm{H}$ & $\mathrm{Up}$ & $\begin{array}{l}\text { Dried root is } \\
\text { boiled and taken to } \\
\text { treat diarrhoea. }\end{array}$ & $\begin{array}{l}\text { Singh et al., } \\
2016\end{array}$ & 1 \\
\hline 11. & Acorus calamus Linn. & Bojho & Araceae & $\mathrm{H}$ & Up & $\begin{array}{l}\text { Rhizome is used to } \\
\text { cure diarrhoea and } \\
\text { dysentery. } \\
\text { Rhizome powder } \\
\text { is used in } \\
\text { indigestion and to } \\
\text { kill worms. }\end{array}$ & $\begin{array}{l}\text { Rana et al., } \\
\text { 2015; Rokaya } \\
\text { et al., 2010; } \\
\text { Shrestha \& } \\
\text { Dhillon, } 2003\end{array}$ & 3 \\
\hline 12. & $\begin{array}{l}\text { Aegle marmelos(L.) Correa ex } \\
\text { Roxb. }\end{array}$ & Bel & Rutaceae & $\mathrm{T}$ & $\begin{array}{l}\mathrm{B}, \mathrm{F}, \\
\mathrm{L}\end{array}$ & $\begin{array}{l}\text { Fruit and bark } \\
\text { juice is given } \\
\text { orally in diarrhoea } \\
\text { and dysentery. } \\
\text { Leaves juice is } \\
\text { given for stomach } \\
\text { disorders, } \\
\text { dysentery and } \\
\text { constipation. }\end{array}$ & $\begin{array}{l}\text { Singh et al., } \\
\text { 2012; Acharya, } \\
\text { 2012; Dhami, } \\
\text { 2008; Acharya } \\
\text { \& Acharya, } \\
\text { 2009; Ambu et } \\
\text { al., 2020; } \\
\text { Bhattarai et al., } \\
\text { 2009 }\end{array}$ & 6 \\
\hline 13. & Ageratum conyzoides L. & Gandheghas & Asteraceae & $\mathrm{H}$ & W & $\begin{array}{l}\text { Plant decoction } \\
\text { mixed with pepper } \\
\text { paste is given to } \\
\text { acute stomachache } \\
\text { and diarrhoea. }\end{array}$ & $\begin{array}{l}\text { Joshi et al., } \\
\text { 2011; Joshi et } \\
\text { al., } 2018\end{array}$ & 2 \\
\hline 14. & $\begin{array}{l}\text { Aleuritopteris bicolor } \\
\text { (Roxb.)Fraser-Jenk. }\end{array}$ & & Pteridaceae & $\mathrm{H}$ & $\mathrm{Wp}$ & $\begin{array}{l}\text { dysentery and } \\
\text { gastritis. }\end{array}$ & $\begin{array}{l}\text { Adhikari et al., } \\
2019\end{array}$ & 1 \\
\hline 15. & Allium carolinianum DC. & ban lasun & Amaryllidaceae & $\mathrm{H}$ & $\mathrm{Bu}$ & $\begin{array}{l}\text { The paste of bulb } \\
\text { is boiled and soup } \\
\text { is used in } \\
\text { indigestion and } \\
\text { stomach disorders, }\end{array}$ & $\begin{array}{l}\text { Rokaya et al., } \\
2010\end{array}$ & 1 \\
\hline 16. & Allium cepa $\mathrm{L}$. & Pyaaj & Amaryllidaceae & $\mathrm{H}$ & $\mathrm{Bu}$ & $\begin{array}{l}\text { The paste of bulb } \\
\text { is boiled and soup } \\
\text { is used for } \\
\text { indigestion and } \\
\text { stomach disorders. }\end{array}$ & $\begin{array}{l}\text { Rokaya et al., } \\
2010\end{array}$ & 1 \\
\hline 17. & Allium fasciculatum Rendle. & & Amaryllidaceae & $\mathrm{H}$ & $\mathrm{Wp}$ & $\begin{array}{l}\text { Whole plant is } \\
\text { grinded and paste } \\
\text { is boiled and used } \\
\text { in stomachache } \\
\text { and diarrhoea. }\end{array}$ & $\begin{array}{l}\text { Rokaya et al., } \\
2010\end{array}$ & 1 \\
\hline 18. & Allium hypsistum Stearn & Chyapi & Amaryllidaceae & $\mathrm{H}$ & $\mathrm{Wp}$ & $\begin{array}{l}\text { The powder of } \\
\text { whole plant is } \\
\text { taken with hot } \\
\text { water for killing } \\
\text { worms in stomach. }\end{array}$ & $\begin{array}{l}\text { Rokaya et al., } \\
2010\end{array}$ & 1 \\
\hline 19. & Allium sativum $\mathrm{L}$. & Lasun & Amaryllidaceae & $\mathrm{H}$ & Up & $\begin{array}{l}\text { Bulb decoction is } \\
\text { given in gastritis, } \\
\text { diarrhoea and } \\
\text { dysentrey. }\end{array}$ & $\begin{array}{l}\text { Adhikari et al., } \\
2019\end{array}$ & 1 \\
\hline
\end{tabular}




\begin{tabular}{|c|c|c|c|c|c|c|c|c|}
\hline 20. & Alnus nepalensis D.Don & Utish & Betulaceae & $\mathrm{T}$ & $\mathrm{B}$ & $\begin{array}{l}\text { Bark decoction is } \\
\text { drunk to treat } \\
\text { gastric problems. }\end{array}$ & $\begin{array}{l}\text { Shrestha \& } \\
\text { Dhillon, } 2003\end{array}$ & 1 \\
\hline 21. & Aloe vera (L.) Burm.f. & Gheukumari & Asphodelaceae & $\mathrm{H}$ & $\mathrm{L}$ & $\begin{array}{l}\text { Leaf pulp is given } \\
\text { orally in stomach } \\
\text { disorders, } \\
\text { constipation and } \\
\text { indigestion. It } \\
\text { increases appetite. }\end{array}$ & $\begin{array}{l}\text { Singh et al., } \\
\text { 2012; Acharya, } \\
\text { 2012; Dhami, } \\
\text { 2008; Ambu et } \\
\text { al., } 2020\end{array}$ & 4 \\
\hline 22. & Alstonia scholaris (L.) R.Br. & & Apocynaceae & $\mathrm{T}$ & $\mathrm{B}$ & $\begin{array}{l}\text { Bark juice is given } \\
\text { in diarrhoea and } \\
\text { dysentery. }\end{array}$ & Dhami, 2008 & 1 \\
\hline 23. & $\begin{array}{l}\text { Alternanthera sessilis (L.) R. Br. } \\
\text { ex DC. }\end{array}$ & & Amaranthaceae & $\mathrm{H}$ & $\mathrm{Wp}$ & $\begin{array}{l}\text { Plant paste is used } \\
\text { in bloody } \\
\text { dysentery. }\end{array}$ & Dhami, 2008 & 1 \\
\hline 24. & Amaranthus caudatus L. & Latte & Amaranthaceae & $\mathrm{H}$ & $\begin{array}{l}\text { Sh, } \\
\text { Up }\end{array}$ & $\begin{array}{l}\text { Tender shoot is } \\
\text { used as vegetable } \\
\text { and used in } \\
\text { diarrhoea and } \\
\text { dysentery. Root } \\
\text { juice is used as } \\
\text { laxative and used } \\
\text { in diarrhoea. }\end{array}$ & $\begin{array}{l}\text { Rokaya et al., } \\
\text { 2010; Malla et } \\
\text { al., } 2015\end{array}$ & 2 \\
\hline 25. & Amaranthus hybridus L. & & Amaranthaceae & I & $\mathrm{Sh}$ & $\begin{array}{l}\text { Tender shoot is } \\
\text { cooked as } \\
\text { vegetables which } \\
\text { improves } \\
\text { digestion. }\end{array}$ & $\begin{array}{l}\text { Rokaya et al., } \\
2010\end{array}$ & 1 \\
\hline 26. & Amaranthus lividus $\mathrm{L}$ & Latte & Amaranthaceae & $\mathrm{H}$ & $\mathrm{Sh}$ & useful in diarrhoea. & 2010 & 1 \\
\hline 27. & Amaranthus spinosus L. & Lunde & Amaranthaceae & $\mathrm{H}$ & Up & $\begin{array}{l}\text { Root juice mixed } \\
\text { with root juice of } \\
\text { Rubus ellipticus is } \\
\text { given to treat } \\
\text { stomach disorders. }\end{array}$ & $\begin{array}{l}\text { Manandhar et } \\
\text { al., 1990; } \\
\text { Dhami, 2008 }\end{array}$ & 1 \\
\hline 28. & Ammomum subulatumRoxb. & Alaichi & Zingiberaceae & $\mathrm{H}$ & $\mathrm{F}$ & $\begin{array}{l}\text { Seeds are taken in } \\
\text { stomachache and } \\
\text { aromatic appetizer. }\end{array}$ & $\begin{array}{l}\text { Rana et al., } \\
2015\end{array}$ & 1 \\
\hline 29. & $\begin{array}{l}\text { Amorphophallus campanulatus } \\
\text { (Roxb.) Blume ex Decne }\end{array}$ & & Araceae & $\mathrm{H}$ & Up & $\begin{array}{l}\text { Cooked corm is } \\
\text { given in dysentery. }\end{array}$ & $\begin{array}{l}\text { Singh et al., } \\
2018\end{array}$ & 1 \\
\hline 30. & $\begin{array}{l}\text { Ampelocissus divaricata (Wall. . } \\
\text { ex M.A.Lawson ) Planch }\end{array}$ & & Vitaceae & $\mathrm{C}$ & $\mathrm{F}$ & $\begin{array}{l}\text { Fruit juice is taken } \\
\text { orally in } \\
\text { indigestion. }\end{array}$ & $\begin{array}{l}\text { Singh et } \\
\text { al. } 2018\end{array}$ & 1 \\
\hline 31. & Anagallis arvensis L. & Armale & Primulaceae & $\mathrm{H}$ & $\mathrm{Wp}$ & $\begin{array}{l}\text { Whole plant is } \\
\text { taken in diarrhoea, } \\
\text { dysentery and } \\
\text { stomachache. }\end{array}$ & Acharya, 2012 & 1 \\
\hline 32. & Ananas comosus (L.) Merr. & Pineapple & Bromeliaceae & $\mathrm{H}$ & $\mathrm{L}$ & $\begin{array}{l}\text { Leaf is orally } \\
\text { given in } \\
\text { constipation.Fresh } \\
\text { leaves are chewed } \\
\text { to expel intesinal } \\
\text { worms. }\end{array}$ & $\begin{array}{l}\text { Singh et al., } \\
2018\end{array}$ & 1 \\
\hline
\end{tabular}




\begin{tabular}{|c|c|c|c|c|c|c|c|c|}
\hline 33. & Anaphalis margaritaceae L. & Bukiphool & Asteraceae & $\mathrm{H}$ & Up & $\begin{array}{l}\text { Root juice is used } \\
\text { in diarrhoea and } \\
\text { dysentery. }\end{array}$ & $\begin{array}{l}\text { Malla et } \\
\text { al.,2015 }\end{array}$ & 1 \\
\hline 34. & Anemone obtusiloba D.Don & & Ranunculaceae & $\mathrm{H}$ & $\mathrm{F}$ & $\begin{array}{l}\text { Seeds are chewed } \\
\text { for indigestion. }\end{array}$ & $\begin{array}{l}\text { Rokaya et al., } \\
2010\end{array}$ & 1 \\
\hline 35. & Anemone polyanthes D.Don & & Ranunculaceae & $\mathrm{H}$ & $\mathrm{F}$ & $\begin{array}{l}\text { Seeds are chewed } \\
\text { for indigestion. }\end{array}$ & $\begin{array}{l}\text { Rokaya et al., } \\
2010\end{array}$ & 1 \\
\hline 36. & $\begin{array}{l}\text { Anemone rivularis Buch.-Ham. } \\
\text { ex DC }\end{array}$ & & Ranunculaceae & $\mathrm{H}$ & $\mathrm{F}$ & $\begin{array}{l}\text { Seeds are chewed } \\
\text { for indigestion. }\end{array}$ & $\begin{array}{l}\text { Rokaya et al., } \\
2010\end{array}$ & 1 \\
\hline 37. & Anemone rupicola Cambess. & & Ranunculaceae & $\mathrm{H}$ & $\mathrm{F}$ & $\begin{array}{l}\text { Seeds are chewed } \\
\text { or powdered and } \\
\text { used in indigestion } \\
\text { and gastritis. }\end{array}$ & $\begin{array}{l}\text { Rokaya et al., } \\
2010\end{array}$ & 1 \\
\hline 38. & Angelica archangelica L. & & Apiaceae & $\mathrm{H}$ & $\mathrm{L}$ & $\begin{array}{l}\text { Leaf decoction is } \\
\text { given to abdominal } \\
\text { pain. }\end{array}$ & $\begin{array}{l}\text { Malla et al., } \\
2015\end{array}$ & 1 \\
\hline 39. & Aralia cachemirica Decne & & Araliaceae & $\mathrm{S}$ & $\mathrm{Up}$ & $\begin{array}{l}\text { Root paste is taken } \\
\text { with hot water for } \\
\text { stomachache and } \\
\text { gatritis. }\end{array}$ & $\begin{array}{l}\text { Singh et al., } \\
2016\end{array}$ & 1 \\
\hline 40. & Araucaria bidwillii Hook. & & Araucariaceae & $\mathrm{T}$ & $\mathrm{B}, \mathrm{L}$ & $\begin{array}{l}\text { tree bark and } \\
\text { leaves are chewed } \\
\text { directly to treat } \\
\text { gastritis and } \\
\text { stomachache. }\end{array}$ & $\begin{array}{l}\text { Sing et al., } \\
2016\end{array}$ & 1 \\
\hline 41. & Arctium lappa L. & Kuro & Asteraceae & $\mathrm{H}$ & F & $\begin{array}{l}\text { seed powder } \\
\text { mixed with water } \\
\text { and infusion is } \\
\text { taken orally in } \\
\text { indigestion. }\end{array}$ & $\begin{array}{l}\text { Rokaya et al., } \\
2010\end{array}$ & 1 \\
\hline 42. & Argemone Mexicana L. & & Papaveraceae & $\mathrm{S}$ & $\mathrm{F}$ & $\begin{array}{l}\text { Seed juice is drunk } \\
\text { in indigestion. }\end{array}$ & $\begin{array}{l}\text { Shrestha \& } \\
\text { Dhillon, } 2003\end{array}$ & 1 \\
\hline 43. & Arisaema jacquemontii Blume. & Bako & Araceae & $\mathrm{H}$ & $\mathrm{L}, \mathrm{Up}$ & $\begin{array}{l}\text { leaves are cooked } \\
\text { as vegetables to } \\
\text { cure stomachache. } \\
\text { Tuber paste is } \\
\text { consumed during } \\
\text { gastritis. }\end{array}$ & $\begin{array}{l}\text { Rokaya et al., } \\
\text { 2010; Singh et } \\
\text { al., } 2016\end{array}$ & 2 \\
\hline 44. & $\begin{array}{l}\text { Arisaema nepenthoides (Wall.) } \\
\text { Mart. }\end{array}$ & Bako & Araceae & $\mathrm{H}$ & $\mathrm{L}$ & $\begin{array}{l}\text { leaves are eaten as } \\
\text { vegetables to cure } \\
\text { stomachache and } \\
\text { constipation. }\end{array}$ & $\begin{array}{l}\text { Rokaya et al., } \\
2010\end{array}$ & 1 \\
\hline 45. & $\begin{array}{l}\text { Arisaema tortuosum (Wall.) } \\
\text { Schott }\end{array}$ & Gaubako & Araceae & $\mathrm{H}$ & $\mathrm{L}, \mathrm{Up}$ & $\begin{array}{l}\text { Leaves and tubers } \\
\text { are used as } \\
\text { vegetables and is } \\
\text { beneficial for } \\
\text { stomachache. }\end{array}$ & $\begin{array}{l}\text { Rokaya et al., } \\
2010\end{array}$ & 1 \\
\hline 46. & Artemisia vulgaris $L$. & Titepati & Asteraceae & $\mathrm{S}$ & $\mathrm{L}$ & $\begin{array}{l}\text { Leaf juice is used } \\
\text { in gastritis. }\end{array}$ & $\begin{array}{l}\text { Acharya, 2012; } \\
\text { Joshi et al., } \\
2018\end{array}$ & 2 \\
\hline 47. & Artocarpus heterophyllus Lam. & Katahar & Moraceae & $\mathrm{T}$ & $\mathrm{B}$ & $\begin{array}{l}\text { Bark juice is used } \\
\text { to treat bloody } \\
\text { dysentery. }\end{array}$ & Dhami, 2008 & 1 \\
\hline
\end{tabular}




\begin{tabular}{|c|c|c|c|c|c|c|c|c|}
\hline 48. & $\begin{array}{l}\text { Asparagus filicinus Buch.-Ham. } \\
\text { Ex D.Don }\end{array}$ & & Asparagaceae & $\mathrm{H}$ & Up & $\begin{array}{l}\text { root decoction or } \\
\text { powder used as } \\
\text { anthelmintic and to } \\
\text { cure diarrhoea. }\end{array}$ & $\begin{array}{l}\text { Rokaya et al., } \\
2010\end{array}$ & 1 \\
\hline 49. & $\begin{array}{l}\text { Astilbe rivularis Buch.-Ham. Ex } \\
\text { D.Don }\end{array}$ & Thulo okhati & Saxifragaceae & $\mathrm{H}$ & Up & $\begin{array}{l}\text { Root juice is used } \\
\text { as apetizer and } \\
\text { taken in diarrhoea } \\
\text { and dysentery. Dry } \\
\text { rootb is directly } \\
\text { chewed during } \\
\text { stomach pain }\end{array}$ & $\begin{array}{l}\text { Acharya, 2012; } \\
\text { Singh et al., } \\
2016\end{array}$ & 2 \\
\hline 50. & Azadirachta indica A.Juss. & Neem & Meliaceae & $\mathrm{T}$ & $\mathrm{B}, \mathrm{L}$ & $\begin{array}{l}\text { Leaf and bark is } \\
\text { used to treat } \\
\text { diarrhoea and } \\
\text { dysentery. Leaf } \\
\text { decoction is } \\
\text { antihelmintic. }\end{array}$ & $\begin{array}{l}\text { Dhami, 2008; } \\
\text { Bhattarai et al., } \\
2009\end{array}$ & 2 \\
\hline 51. & Basella alba L. & & Basellaceae & $\mathrm{C}$ & $\mathrm{L}$ & $\begin{array}{l}\text { Leaves decoction } \\
\text { is given in } \\
\text { constipation. }\end{array}$ & $\begin{array}{l}\text { Singh et al., } \\
2018\end{array}$ & 1 \\
\hline 52. & Bauhinia purpurea $\mathrm{L}$. & Tanki & Fabaceae & $\mathrm{T}$ & $\mathrm{Fl}$ & $\begin{array}{l}\text { Mature flower and } \\
\text { buds are taken to } \\
\text { treat stomachache } \\
\text { and diarrhoea. }\end{array}$ & $\begin{array}{l}\text { Joshi et al., } \\
2011 .\end{array}$ & 1 \\
\hline 53. & Bauhinia vahlii Wight \& Arn. & Bhorla & Fabaceae & $\mathrm{S}$ & $\begin{array}{l}\mathrm{B}, \mathrm{F}, \\
\mathrm{Up}\end{array}$ & $\begin{array}{l}\text { Bark juice is taken } \\
\text { to cure diarrhoea } \\
\text { and dysentery. } \\
\text { Root juice is given } \\
\text { in dysentery. } \\
\text { Roasted or boiled } \\
\text { seeds are good in } \\
\text { indigestion and } \\
\text { acidity. }\end{array}$ & $\begin{array}{l}\text { Acharya, 2012; } \\
\text { Dhami, } 2008\end{array}$ & 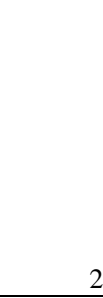 \\
\hline 54. & Bauhinia Variegata L. & Koiralo & Fabaceae & $\mathrm{T}$ & $\mathrm{B}, \mathrm{Fl}$ & $\begin{array}{l}\text { Bark decoction } \\
\text { and flower juice } \\
\text { are given in } \\
\text { diarrhoea, } \\
\text { dysentery and } \\
\text { indigestion. }\end{array}$ & $\begin{array}{l}\text { Singh et al., } \\
\text { 2012; Acharya, } \\
\text { 2012; Dhami, } \\
\text { 2008; Acharya } \\
\text { \& Acharya, } \\
2009\end{array}$ & 4 \\
\hline 55. & Begonia picta $\mathrm{Sm}$. & & Begoniaceae & $\mathrm{H}$ & Up & $\begin{array}{l}\text { Root infusion is } \\
\text { taken orally to cure } \\
\text { constipation. }\end{array}$ & $\begin{array}{l}\text { Rana et al., } \\
2015\end{array}$ & 1 \\
\hline 56. & $\begin{array}{l}\text { Benincasa hispida (Thunb.) } \\
\text { Cogn. }\end{array}$ & Kubindo & Cucurbitaceae & $\mathrm{C}$ & $\mathrm{L}$ & $\begin{array}{l}\text { Leaf juice is } \\
\text { recommended for } \\
\text { colic pain. }\end{array}$ & $\begin{array}{l}\text { Malla et al., } \\
2015\end{array}$ & 1 \\
\hline 57. & Berberis aristata DC. & Chutro & Berberidaceae & $\mathrm{S}$ & $\begin{array}{l}\mathrm{F}, \mathrm{L} \\
\mathrm{Up}\end{array}$ & $\begin{array}{l}\text { Root juice is } \\
\text { recommended for } \\
\text { dysentery. Fruit } \\
\text { and leaf juice is } \\
\text { used to treat } \\
\text { diarrhoea and } \\
\text { dysentery. }\end{array}$ & $\begin{array}{l}\text { Malla et al., } \\
\text { 2015; Kunwar } \\
\text { \& Adhikari, } \\
2005 .\end{array}$ & 2 \\
\hline 58. & Berberis lycium Royle & & Berberidaceae & $\mathrm{S}$ & $\begin{array}{l}\mathrm{B}, \mathrm{F} \text {, } \\
\mathrm{L}\end{array}$ & $\begin{array}{l}\text { Infusion of fruits, } \\
\text { barks and leaves } \\
\text { are used to cure } \\
\text { diarrhoea. }\end{array}$ & $\begin{array}{l}\text { Rokaya et al., } \\
2010\end{array}$ & \\
\hline 59. & Berberis mucrifolia Ahrendt & & Berberidaceae & $\mathrm{S}$ & Wp & $\begin{array}{l}\text { Infusion of whole } \\
\text { plant is given } \\
\text { orally to treat } \\
\text { diarrhoea and } \\
\text { dysentery. }\end{array}$ & $\begin{array}{l}\text { Rokaya et al., } \\
2010\end{array}$ & 1 \\
\hline
\end{tabular}




\begin{tabular}{|c|c|c|c|c|c|c|c|c|}
\hline 60. & Berginia ciliata (Haw.) Sternb. & Pakhambhed & Saxifragaceae & $\mathrm{H}$ & $\mathrm{Up}$ & $\begin{array}{l}\text { Rhizome is } \\
\text { powered and given } \\
\text { to treat swollen } \\
\text { stomach. Root } \\
\text { powder is given in } \\
\text { diarrhoea, } \\
\text { dysentery and } \\
\text { vomitting }\end{array}$ & $\begin{array}{l}\text { Rana et al., } \\
\text { 2015; Rokaya } \\
\text { et al., 2010; } \\
\text { Limbu \& Rai, } \\
\text { 2013; Kunwar } \\
\text { \& Adhikari, } \\
\text { 2005; Ambu et } \\
\text { al., 2020 }\end{array}$ & 6 \\
\hline 61. & $\begin{array}{l}\text { Calotropis gigantean ( L.) } \\
\text { Dryand. }\end{array}$ & Chyauphool & Polygonaceae & $\mathrm{H}$ & Wp & $\begin{array}{l}\text { The decoction of } \\
\text { plant is given in } \\
\text { diarrhoea and } \\
\text { dysentery. }\end{array}$ & $\begin{array}{l}\text { Malla et al., } \\
2015\end{array}$ & 1 \\
\hline 62. & Boehmeria platyphylla d.Don & Kamleghans & Urticaceae & $\mathrm{H}$ & $\mathrm{L}$ & $\begin{array}{l}\text { Decoction of leaf } \\
\text { is given in } \\
\text { diarrhoea and } \\
\text { dysentery. }\end{array}$ & $\begin{array}{l}\text { Malla et al., } \\
2015\end{array}$ & 1 \\
\hline 63. & Bombax ceiba $\mathrm{L}$. & Simal & Malvaceae & $\mathrm{T}$ & $\mathrm{B}$ & $\begin{array}{l}\text { Bark powder is } \\
\text { given in gastric } \\
\text { disorder and } \\
\text { gastric. Plant juice } \\
\text { is is used in } \\
\text { indigestion, } \\
\text { diarrhoea and } \\
\text { dysentery. }\end{array}$ & $\begin{array}{l}\text { Rana et al., } \\
\text { 2015; Dhami, } \\
\text { 2008; Bhattarai } \\
\text { et al., 2009; } \\
\text { Rai, 2004. }\end{array}$ & 4 \\
\hline 64. & Bridelia retusa (L.) A. Juss. & Gayo & Phyllanthaceae & $\mathrm{T}$ & $\mathrm{B}$ & $\begin{array}{l}\text { Bark extract is } \\
\text { used to treat } \\
\text { diarrhoea. }\end{array}$ & $\begin{array}{l}\text { Limbu \& Rai, } \\
2013\end{array}$ & 1 \\
\hline 65. & $\begin{array}{l}\text { Bryophyllum pinnatum (Lam.) } \\
\text { Oken. }\end{array}$ & Ajambari & Crassulaceae & $\mathrm{H}$ & $\mathrm{L}$ & $\begin{array}{l}\text { Leaf juice or } \\
\text { decoction is given } \\
\text { in dysentery and } \\
\text { stomachache. }\end{array}$ & $\begin{array}{l}\text { Malla et al., } \\
\text { 2015; Joshi et } \\
\text { al., } 2018\end{array}$ & 2 \\
\hline 66. & Callicarpa arborea Roxb. & Masgedi & Verbinaceae & $\mathrm{T}$ & $\mathrm{B}$ & $\begin{array}{l}\text { Bark juice is given } \\
\text { in indigestion. }\end{array}$ & $\begin{array}{l}\text { Malla et al., } \\
2015\end{array}$ & 1 \\
\hline 67. & Callicarpa macrophylla Vahl. & 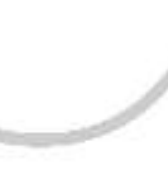 & Verbenaceae & $\mathrm{S}$ & $\begin{array}{l}\text { Bud, } \\
\text { Up }\end{array}$ & $\begin{array}{l}\text { Root juice is given } \\
\text { in indigestion. } \\
\text { Young bud paste is } \\
\text { drunk in } \\
\text { stomachache. }\end{array}$ & $\begin{array}{l}\text { Dhami, 2008; } \\
\text { Manandhar, } \\
1987\end{array}$ & 2 \\
\hline 68. & Calotropis gigantea L. Dryand. & & Asclepladaceae & $\mathrm{T}$ & $\mathrm{B}$ & $\begin{array}{l}\text { Bark juice is used } \\
\text { to treat diarrhoea } \\
\text { and dysentery. }\end{array}$ & Dhami, 2008 & 1 \\
\hline 69. & Campanula pallida Wall. & Ganubuti & Campanulaceae & $\mathrm{C}$ & $\mathrm{Up}$ & $\begin{array}{l}\text { roots are used to } \\
\text { treat diarrhoea and } \\
\text { dysentery. }\end{array}$ & $\begin{array}{l}\text { Manandhar, } \\
\text { 1990; Malla et } \\
\text { al., 2015 }\end{array}$ & 2 \\
\hline 70. & Cannabis sativa $\mathrm{L}$. & Ganja & Cannabaceae & $\mathrm{H}$ & $\begin{array}{l}\mathrm{F}, \mathrm{Fl}, \\
\mathrm{L}\end{array}$ & $\begin{array}{l}\text { Juice of flowers } \\
\text { and fruits and } \\
\text { seeds are taken } \\
\text { orally for } \\
\text { diarrhoea,dysenter } \\
\text { y and constipation. } \\
\text { And also acts as } \\
\text { apetizer. Leaf is } \\
\text { chewed and } \\
\text { swallowed in } \\
\text { indigestion. }\end{array}$ & $\begin{array}{l}\text { Rokaya et al., } \\
\text { 2010. Joshi et } \\
\text { al., } 2011 . \\
\text { Shrestha \& } \\
\text { Dhillon, 2003. } \\
\text { Manandhar, } \\
\text { 1987; Bhattarai } \\
\text { et al., 2009 }\end{array}$ & 6 \\
\hline 71. & Capparis zeylanica $\mathrm{L}$. & Baghmukhe & Capparaceae & $\mathrm{C}$ & $\mathrm{Up}$ & $\begin{array}{l}\text { Root bark paste is } \\
\text { orally given in } \\
\text { stomachache. }\end{array}$ & $\begin{array}{l}\text { Singh et al., } \\
2018\end{array}$ & 1 \\
\hline 72. & Careya arborea Roxb. & & Lecythidaceae & $\mathrm{T}$ & $\mathrm{B}, \mathrm{F}$ & $\begin{array}{l}\text { Bark and fruit } \\
\text { juice is used as } \\
\text { medicine in } \\
\text { dysentery. }\end{array}$ & $\begin{array}{l}\text { Acharya and } \\
\text { Acharya, } 2009\end{array}$ & 1 \\
\hline
\end{tabular}




\begin{tabular}{|c|c|c|c|c|c|c|c|c|}
\hline 73. & Carica papaya $\mathrm{L}$. & Mewa & Caricaceae & $\mathrm{S}$ & $\mathrm{La}$ & $\begin{array}{l}\text { Milky latex is } \\
\text { given in dysentery. }\end{array}$ & $\begin{array}{l}\text { Singh et al., } \\
\text { 2012; Dhami, } \\
2008\end{array}$ & 2 \\
\hline 74. & Carum carvi $\mathrm{L}$. & Bhotejeera & Apiaceae & $\mathrm{H}$ & $\mathrm{F}$ & $\begin{array}{l}\text { Fruits are chewed } \\
\text { to increase apetite } \\
\text { and to cure } \\
\text { stomach problems. }\end{array}$ & $\begin{array}{l}\text { Rokaya et al., } \\
2010\end{array}$ & 1 \\
\hline 75. & Cassia agnes (de Wit) Brenan & & Fabaceae & $\mathrm{S}$ & $\mathrm{Up}$ & $\begin{array}{l}\text { Root decoction } \\
\text { with salt is taken } \\
\text { for indigestion. }\end{array}$ & $\begin{array}{l}\text { Singh et al. } \\
2018\end{array}$ & 1 \\
\hline 76. & Cassia fistula $\mathrm{L}$. & Rajbrikshya & Fabaceae & $\mathrm{T}$ & $\mathrm{F}$ & $\begin{array}{l}\text { Fruit pulp is used } \\
\text { to treat indigestion. } \\
\text { Seed is used to } \\
\text { treat constipation, } \\
\text { diarrhoea, } \\
\text { dysentery and } \\
\text { stomachache. }\end{array}$ & $\begin{array}{l}\text { Dhami, 2008; } \\
\text { Limbu \& Rai, } \\
\text { 2013; Bhattarai } \\
\text { et al., } 2009\end{array}$ & 3 \\
\hline 77. & $\begin{array}{l}\text { Cassia tomentella } \\
\text { (Benth.)Domin }\end{array}$ & & Fabaceae & $\mathrm{T}$ & $\mathrm{F}$ & $\begin{array}{l}\text { Seed paste is used } \\
\text { as antihelmintic. }\end{array}$ & Dhami, 2008 & 1 \\
\hline 78. & Castanopsis indica (Roxb.) Miq. & Katush & Fagaceae & $\mathrm{T}$ & $\mathrm{L}$ & $\begin{array}{l}\text { Leaf or bud juice } \\
\text { is taken for } \\
\text { stomach disorders. }\end{array}$ & $\begin{array}{l}\text { Manandhar, } \\
1990\end{array}$ & 1 \\
\hline 79. & $\begin{array}{l}\text { Catunaregam spinosa (Thunb.) } \\
\text { Tirveng. }\end{array}$ & & Rubiaceae & $\mathrm{T}$ & $\mathrm{B}$ & $\begin{array}{l}\text { Bark powder is } \\
\text { used to treat } \\
\text { gastritis and } \\
\text { stomachache. }\end{array}$ & $\begin{array}{l}\text { Bhattarai et al., } \\
2009\end{array}$ & 1 \\
\hline 80. & Cautleya spicata (Sm.) Baker & Banbesar & Zingiberaceae & $\mathrm{H}$ & Up & $\begin{array}{l}\text { Rhizome juice is } \\
\text { given to treat } \\
\text { stomach disorders } \\
\text { and constipation. }\end{array}$ & $\begin{array}{l}\text { Manandhar, } \\
\text { 1990; Ambu et } \\
\text { al., } 2020\end{array}$ & 2 \\
\hline 81. & $\begin{array}{l}\text { Cedrus deodara (Roxb. Ex } \\
\text { D.Don) Hook.f. }\end{array}$ & Debdar & Pinaceae & $\mathrm{T}$ & $\mathrm{B}$ & $\begin{array}{l}\text { Bark decoction is } \\
\text { used to treat } \\
\text { diarrhoea and } \\
\text { dysentery. }\end{array}$ & $\begin{array}{l}\text { kunwar \& } \\
\text { Adhikari, } 2005\end{array}$ & 1 \\
\hline 82. & Celosia argenteaL. & & Amaranthaceae & $\mathrm{H}$ & $\mathrm{L}$ & $\begin{array}{l}\text { Leaf juice is used } \\
\text { to treat diarrhoea } \\
\text { and dysentery. }\end{array}$ & $\begin{array}{l}\text { kunwar \& } \\
\text { Adhikari, } 2005\end{array}$ & 1 \\
\hline 83. & Centella asiatica (L.) Urb. & Ghodtapre & Apiaceae & $\mathrm{H}$ & $\mathrm{Wp}$ & $\begin{array}{l}\text { Leaf juice is given } \\
\text { orally in } \\
\text { indigestion. Plant } \\
\text { decoction is given } \\
\text { in indigestion and } \\
\text { dysentery. }\end{array}$ & $\begin{array}{l}\text { Adhikari, et al., } \\
\text { 2019; Singh et } \\
\text { al., 2012; Malla } \\
\text { et al., 2015; } \\
\text { Kunwar \& } \\
\text { Adhikari, } 2005\end{array}$ & 4 \\
\hline 84. & Chamabainia cuspidata Wight. & & Urticaceae & Herb & $\mathrm{Wp}$ & $\begin{array}{l}\text { Plant juice is } \\
\text { given in stomach } \\
\text { disorders. }\end{array}$ & $\begin{array}{l}\text { Manandhar, } \\
1990\end{array}$ & 1 \\
\hline 85. & $\begin{array}{l}\text { Cheilanthes albomarginata } \\
\text { C..B.Clarke }\end{array}$ & & Pteridaceae & Herb & $\mathrm{Wp}$ & $\begin{array}{l}\text { Plant is crushed, } \\
\text { filtered and taken } \\
\text { to dysentery and } \\
\text { stomach disorders. }\end{array}$ & $\begin{array}{l}\text { Rana et al., } \\
\text { 2015; Joshi et } \\
\text { al., } 2011 .\end{array}$ & 2 \\
\hline
\end{tabular}




\begin{tabular}{|c|c|c|c|c|c|c|c|c|}
\hline 86. & Chenopodium album L. & Bethe & Chenopodiaceae & $\mathrm{H}$ & F, Sh & $\begin{array}{l}\text { Seed powder is } \\
\text { used to make bread } \\
\text { and taken in } \\
\text { abdominal pain. } \\
\text { Tender shoot \& } \\
\text { flower juice is } \\
\text { given orally to kill } \\
\text { worms, gastritis } \\
\text { and remove } \\
\text { constipation. }\end{array}$ & $\begin{array}{l}\text { Adhikari, et al., } \\
\text { 2019; Rana } \text { et } \\
\text { al., 2015; } \\
\text { Singh et al., } \\
\text { 2012; Rokaya } \\
\text { et al., 2010; } \\
\text { Joshi et al., } \\
2011\end{array}$ & 6 \\
\hline 87. & Chenopodium murale L. & Bhatebethe & Chenopodiaceae & $\mathrm{H}$ & $\mathrm{L}$ & $\begin{array}{l}\text { Fresh leaf is used } \\
\text { to treat diarrhoea } \\
\text { and dysentery. }\end{array}$ & $\begin{array}{l}\text { kunwar \& } \\
\text { Adhikari, } 2005\end{array}$ & 1 \\
\hline 88. & $\begin{array}{l}\text { Choerospondias axillaris (Roxb.) } \\
\text { Brutt \& Hill }\end{array}$ & Lapsi & Anacardiaceae & $\mathrm{T}$ & $\mathrm{B}$ & $\begin{array}{l}\text { Bark is usually } \\
\text { taken for } \\
\text { dysentery. }\end{array}$ & $\begin{array}{l}\text { Rana et al., } \\
2015\end{array}$ & 1 \\
\hline 89. & $\begin{array}{l}\text { Chonemorpha fragrans (Moon.) } \\
\text { Alston }\end{array}$ & & Apocynaceae & $\mathrm{H}$ & Up & $\begin{array}{l}\text { Root decoction is } \\
\text { given in } \\
\text { constipation. }\end{array}$ & $\begin{array}{l}\text { Malla et } \\
\text { al.,2015 }\end{array}$ & 1 \\
\hline 90. & $\begin{array}{l}\text { Cinnamomum tamala (Buch.- } \\
\text { Ham.) Nees. }\end{array}$ & Tejpat & Laur & $\mathrm{T}$ & $\mathrm{B}, \mathrm{L}$ & $\begin{array}{l}\text { Leaf and bark is } \\
\text { used to treat } \\
\text { stomachache, } \\
\text { dysentery, gastritis } \\
\text { and to increase } \\
\text { apetite. }\end{array}$ & $\begin{array}{l}\text { Adhikari et al., } \\
\text { 2019; Acharya, } \\
\text { 2012; Joshi et } \\
\text { al., 2018; } \\
\text { Singh } \text { et al., } \\
\text { 2016; Kunwar } \\
\text { \& Adhikari, } \\
\text { 2005; Rai, } \\
\text { 2004. }\end{array}$ & 6 \\
\hline 91. & Cissampelos pareira $\mathrm{L}$. & Batulopate & Menispermaceae & $\mathrm{C}$ & $\begin{array}{l}\text { Up, } \\
\text { Wp }\end{array}$ & $\begin{array}{l}\text { Bulb is eaten to } \\
\text { clear stools. Plant } \\
\text { juice is given in } \\
\text { indigestion and to } \\
\text { kill } \\
\text { intestinalworms, } \\
\text { diarrhoea and } \\
\text { dysentery. }\end{array}$ & $\begin{array}{l}\text { Manandhar, } \\
\text { 1990; Malla et } \\
\text { al., 2015; } \\
\text { Acharya, 2012; } \\
\text { Dhami, 2008 }\end{array}$ & 4 \\
\hline 92. & Citrus lemon (L.) Burm.f. & & Rutaceae & $\mathrm{S}$ & $\mathrm{L}$ & $\begin{array}{l}\text { Fresh leaves are } \\
\text { chewed to expel } \\
\text { intesinal worms. }\end{array}$ & $\begin{array}{l}\text { Singh et al., } \\
2012\end{array}$ & 1 \\
\hline 93. & Citrus medica L. & Bimiro & Rutaceae & $\mathrm{T}$ & F, Up & $\begin{array}{l}\text { Root and fruit } \\
\text { juice is used to kill } \\
\text { intestinal worms. }\end{array}$ & $\begin{array}{l}\text { Adhikari et al., } \\
2019\end{array}$ & 1 \\
\hline 94. & Clitoria ternatea $\mathrm{L}$. & & Fabaceae & $\mathrm{S}$ & $\mathrm{L}, \mathrm{Up}$ & $\begin{array}{l}\text { Leaves and root is } \\
\text { given for } \\
\text { dysentery. }\end{array}$ & $\begin{array}{l}\text { Singh } \text { et } \\
\text { al.,2018 }\end{array}$ & 1 \\
\hline 95. & Coix lacryma-Jobi L. & Bhirkaunle & Poaceae & $\mathrm{H}$ & Up & $\begin{array}{l}\text { Root is chewed to } \\
\text { kill intestinal } \\
\text { worms. }\end{array}$ & $\begin{array}{l}\text { Joshi et al., } \\
2011 .\end{array}$ & 1 \\
\hline 96. & Colacasia esculenta (L.)Schott. & Karkalo & Araceae & $\mathrm{H}$ & Up & $\begin{array}{l}\text { Boiled corm is } \\
\text { used to cure } \\
\text { constipation. }\end{array}$ & $\begin{array}{l}\text { Adhikari et al., } \\
\text { 2019; Ambu et } \\
\text { al., } 2020\end{array}$ & 2 \\
\hline 97. & Colebrookea oppositifolia Sm. & Dhursel & Lamiaceae & $\mathrm{S}$ & $\mathrm{L}$ & $\begin{array}{l}\text { Leaf juice is used } \\
\text { as an anthelmintic. }\end{array}$ & $\begin{array}{l}\text { Manandhar, } \\
1990\end{array}$ & 1 \\
\hline 98. & Commelina maculata Edgew. & Kane & Commelinaceae & $\mathrm{H}$ & $\mathrm{L}$ & $\begin{array}{l}\text { Leaf juice is is } \\
\text { given for apetite } \\
\text { and gastritis. }\end{array}$ & $\begin{array}{l}\text { Malla et al., } \\
2015\end{array}$ & 1 \\
\hline 99. & Commelina paludosa Blume & Kanejhar & Commelinaceae & $\mathrm{H}$ & Up & $\begin{array}{l}\text { Root juice is given } \\
\text { in indigestion. }\end{array}$ & $\begin{array}{l}\text { Singh et al., } \\
2018\end{array}$ & 1 \\
\hline
\end{tabular}




\begin{tabular}{|c|c|c|c|c|c|c|c|c|}
\hline 100. & Coriandrum sativum $\mathrm{L}$. & Dhaniya & Apiaceae & $\mathrm{H}$ & $\mathrm{L}$ & $\begin{array}{l}\text { Green leaves are } \\
\text { used in the } \\
\text { preparation of soft } \\
\text { drink and given in } \\
\text { stomachache. }\end{array}$ & $\begin{array}{l}\text { Singh et al., } \\
2012\end{array}$ & 1 \\
\hline 101. & Coriaria nepalensis Wall. & Machhaino & Coriariaceae & $\mathrm{S}$ & $\mathrm{F}$ & $\begin{array}{l}\text { Ripe fruits and } \\
\text { plant njuice is } \\
\text { taken to treat } \\
\text { indigestion. }\end{array}$ & $\begin{array}{l}\text { Ambu et al., } \\
2020\end{array}$ & 1 \\
\hline 102. & $\begin{array}{l}\text { Crassocephalum crepidioides } \\
\text { (Benth.) S. Moore }\end{array}$ & Namlejhar & Asteraceae & $\mathrm{H}$ & $\mathrm{Wp}$ & $\begin{array}{l}\text { Plant juice is given } \\
\text { to cure diarrehoea. }\end{array}$ & $\begin{array}{l}\text { Manandhar, } \\
1990\end{array}$ & 1 \\
\hline 103. & $\begin{array}{l}\text { Crateva unilocularis Buch.- } \\
\text { Ham. }\end{array}$ & Siplican & Capparaceae & $\mathrm{T}$ & $\mathrm{Sh}$ & $\begin{array}{l}\text { Shoot is used to } \\
\text { treat inntestinal } \\
\text { worms. Young } \\
\text { shoots are boiled } \\
\text { and eaten in } \\
\text { stomach pain. }\end{array}$ & $\begin{array}{l}\text { Acharya, 2012; } \\
\text { Ambu et al., } \\
2020\end{array}$ & 2 \\
\hline 104. & Cucurbita maxima D.Don & Pharsi & Cucurbitaceae & $\mathrm{C}$ & $\mathrm{Sh}$ & $\begin{array}{l}\text { Tender shoots cure } \\
\text { constipation }\end{array}$ & $\begin{array}{l}\text { Rokaya et al., } \\
2010\end{array}$ & 1 \\
\hline 105. & Curculigo orchioides Gaertn. & & Hypoxidaceae & $\mathrm{H}$ & Up & $\begin{array}{l}\text { Rhizome is } \\
\text { chewed raw and } \\
\text { swallowed in } \\
\text { dysentery }\end{array}$ & $\begin{array}{l}\text { Shrestha \& } \\
\text { Dhillon, 2003 }\end{array}$ & 1 \\
\hline 106. & Curcuma angustifolia Roxb. & Haledo & Zizingiberaceae & $\mathrm{H}$ & Up & $\begin{array}{l}\text { Root paste is taken } \\
\text { with hot water for } \\
\text { stomachache. }\end{array}$ & $\begin{array}{l}\text { Rokaya et al., } \\
2010\end{array}$ & 1 \\
\hline 107. & Curcuma caesia Roxb. & & Zizingiberaceae & $\mathrm{H}$ & Up & $\begin{array}{l}\text { It is used in } \\
\text { constipation. }\end{array}$ & $\begin{array}{l}\text { Ambu et al., } \\
2020\end{array}$ & 1 \\
\hline 108. & Curcuma longa L. & Besar & Zizingiberaceae & $\mathrm{H}$ & Up & $\begin{array}{l}\text { Fresh rhizome } \\
\text { juice is } \\
\text { anthelmintic. }\end{array}$ & $\begin{array}{l}\text { Singh et al., } \\
2012\end{array}$ & 1 \\
\hline 109. & $\begin{array}{l}\text { Curcuma zedoaria(Christm.) } \\
\text { Roscoe }\end{array}$ & + & Zingiberaceae & $\mathrm{H}$ & $\mathrm{Wp}$ & $\begin{array}{l}\text { Whole plant is } \\
\text { used in stomach } \\
\text { problem. }\end{array}$ & Acharya, 2012 & 1 \\
\hline 110. & Cuscuta reflexa Roxb. & Aakashbeli & Convolvulaceae & $\mathrm{C}$ & Wp & $\begin{array}{l}\text { Plant paste is } \\
\text { applied externally } \\
\text { to treat } \\
\text { stomachache. }\end{array}$ & $\begin{array}{l}\text { Singh et al., } \\
2012\end{array}$ & 1 \\
\hline 111. & Cyathula tomentosa (Roth) Moq. & & Amaranthaceae & $\mathrm{H}$ & Up & $\begin{array}{l}\text { Root juice is used } \\
\text { to treat } \\
\text { constipation. }\end{array}$ & $\begin{array}{l}\text { Rokaya et al., } \\
2010\end{array}$ & 1 \\
\hline 112. & $\begin{array}{l}\text { Cymbopogon flexuosus (Nees. Ex } \\
\text { Steud.) W.Watson }\end{array}$ & Lemongrass & Poaceae & $\mathrm{H}$ & $\mathrm{L}$ & $\begin{array}{l}\text { Leaf decoction is } \\
\text { given in diarrhoea. }\end{array}$ & $\begin{array}{l}\text { Malla et al., } \\
2015\end{array}$ & 1 \\
\hline 113. & 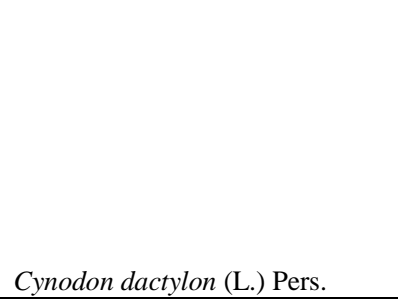 & Dubo & Poaceae & $\mathrm{H}$ & $\mathrm{Wp}$ & $\begin{array}{l}\text { Plant juice is taken } \\
\text { for gastric } \\
\text { disorders and to } \\
\text { kill intestinal } \\
\text { worms, root } \\
\text { infusion with } \\
\text { sugaris given } \\
\text { orally in } \\
\text { indigestion. }\end{array}$ & $\begin{array}{l}\text { Adhikari et al., } \\
\text { 2019; Rana et } \\
\text { al., 2015; } \\
\text { Singh et al., } \\
\text { 2012; Acharya, } \\
\text { 2012; Bhattarai } \\
\text { et al., } 2009\end{array}$ & 6 \\
\hline
\end{tabular}




\begin{tabular}{|c|c|c|c|c|c|c|c|c|}
\hline 114. & Cyperus rotundus $\mathrm{L}$. & Mothe & Cyperaceae & $\mathrm{H}$ & $\mathrm{L}, \mathrm{Up}$ & $\begin{array}{l}\text { Tuber infusion } \\
\text { with sugar/salt is } \\
\text { given orally in } \\
\text { diarrhoea, } \\
\text { dysentery, } \\
\text { indigestion and } \\
\text { stomach pain. }\end{array}$ & $\begin{array}{l}\text { Singh et al., } \\
\text { 2012; Joshi et } \\
\text { al.,2011; } \\
\text { Acharya, 2012; } \\
\text { Dhami, 2008; } \\
\text { Acharya and } \\
\text { Acharya, 2009; } \\
\text { Bhattarai et al., } \\
2009\end{array}$ & 6 \\
\hline 115. & $\begin{array}{l}\text { Dactylicapnos scandens (D.Don) } \\
\text { Hutch. }\end{array}$ & Pahelephul & Papaveraceae & $\mathrm{H}$ & Up & $\begin{array}{l}\text { Root decoction is } \\
\text { used for dysentery. }\end{array}$ & $\begin{array}{l}\text { Malla et al., } \\
2015\end{array}$ & 1 \\
\hline 116. & $\begin{array}{l}\text { Dactylorhiza hatagirea (D.Don) } \\
\text { Soo }\end{array}$ & Panchaunle & Orchidaceae & $\mathrm{H}$ & $\mathrm{Up}$ & $\begin{array}{l}\text { Dry or fresh root is } \\
\text { consumed with hot } \\
\text { water or milk to } \\
\text { treat gastritis and } \\
\text { intestinal ulcer. }\end{array}$ & $\begin{array}{l}\text { Rokaya et al., } \\
\text { 2010; Kunwar } \\
\text { \& Adhikari, } \\
2005\end{array}$ & 2 \\
\hline 117. & Dalbergia sissoo Roxb. ex DC. & Sisoo & Fabaceae & $\mathrm{T}$ & $\mathrm{B}, \mathrm{L}$ & $\begin{array}{l}\text { bark and leaf juice } \\
\text { are given orally in } \\
\text { diarrhoea, } \\
\text { dysentery and } \\
\text { anthelmintic. }\end{array}$ & $\begin{array}{l}\text { Singh et al., } \\
2012\end{array}$ & 1 \\
\hline 118. & $\begin{array}{l}\text { Daphne bholua Buch.-Ham. Ex } \\
\text { D.Don }\end{array}$ & Lokhta & Thymelaecaceae & $\mathrm{S}$ & $\mathrm{Up}$ & $\begin{array}{l}\text { Root powder with } \\
\text { water or root juice } \\
\text { is consumed } \\
\text { during } \\
\text { stomachache. }\end{array}$ & $\begin{array}{l}\text { Singh et al., } \\
\text { 2016; Shrestha } \\
\text { \& Dhillon, } \\
2003\end{array}$ & 2 \\
\hline 119. & Delphinium grandiflorum L. & & Ranunculaceae & $\mathrm{H}$ & $\mathrm{F}, \mathrm{Fl}$ & $\begin{array}{l}\text { Leaves, flowers } \\
\text { and fruits are } \\
\text { crushed and } \\
\text { consumed to loss } \\
\text { of apetite and } \\
\text { stomach problem. }\end{array}$ & $\begin{array}{l}\text { Rokaya et al., } \\
2010\end{array}$ & 1 \\
\hline 120. & Delphinium himalayae Munz & Atish & Ranunculaceae & $\mathrm{H}$ & Wp & $\begin{array}{l}\text { Powder or paste of } \\
\text { whole plant is used } \\
\text { to treat diarrhoea. }\end{array}$ & $\begin{array}{l}\text { Rokaya et al., } \\
2010\end{array}$ & 1 \\
\hline 121. & Desmodium gangeticum (L.) DC. & Kachaurijhar & Fabaceae & $\mathrm{H}$ & Up & $\begin{array}{l}\text { Root juice is given } \\
\text { in diarrhoea. }\end{array}$ & $\begin{array}{l}\text { Singh et al., } \\
2018\end{array}$ & 1 \\
\hline 122. & $\begin{array}{l}\text { Desmodium heterocarpon (L.) } \\
\text { DC. }\end{array}$ & Bangahat & Fabaceae & $\mathrm{H}$ & Up & $\begin{array}{l}\text { Root juice is given } \\
\text { in diarrhoea. }\end{array}$ & $\begin{array}{l}\text { Malla et al., } \\
2015\end{array}$ & 1 \\
\hline 123. & Desmodium multiflorum DC. & Bakhreghans & Fabaceae & $\mathrm{H}$ & $\mathrm{Up}$ & $\begin{array}{l}\text { Root powder } \\
\text { mixed with boiled } \\
\text { water and given in } \\
\text { indigestion. }\end{array}$ & $\begin{array}{l}\text { Malla et al., } \\
2015\end{array}$ & 1 \\
\hline 124. & $\begin{array}{l}\text { Desmostachya bipinnata (L.) } \\
\text { Stapf. }\end{array}$ & Kush & Poaceae & $\mathrm{H}$ & Up & $\begin{array}{l}\text { Root juice is given } \\
\text { in indigestion and } \\
\text { dysentery. }\end{array}$ & $\begin{array}{l}\text { Malla et al., } \\
2015\end{array}$ & 1 \\
\hline 125. & Dioscorea bulbifera L. & Gittha & Dioscoreaceae & $\mathrm{C}$ & Bul & $\begin{array}{l}\text { Boiled bulbil is } \\
\text { used to treat } \\
\text { stomach pain, } \\
\text { intestinal worms } \\
\text { and gastritis. }\end{array}$ & Acharya, 2012 & 1 \\
\hline 126. & $\begin{array}{l}\text { Dioscorea deltoidea Wall. Ex } \\
\text { Griseb }\end{array}$ & Githa & Dioscoreaceae & $\mathrm{C}$ & Bul & $\begin{array}{l}\text { Boiled bulbil is } \\
\text { used to treat } \\
\text { stomach pain. }\end{array}$ & $\begin{array}{l}\text { Acharya and } \\
\text { Acharya, } 2009\end{array}$ & 1 \\
\hline
\end{tabular}




\begin{tabular}{|c|c|c|c|c|c|c|c|c|}
\hline 127. & Dioscorea pentaphylla L. & Bhyakur & Dioscoreaceae & $\mathrm{C}$ & $\mathrm{Up}$ & $\begin{array}{l}\text { Boiled rhizome is } \\
\text { given in abdominal } \\
\text { pain. }\end{array}$ & $\begin{array}{l}\text { Adhikari et al., } \\
\text { 2019; Singh } \text { et } \\
\text { al., } 2012\end{array}$ & 2 \\
\hline 128. & $\begin{array}{l}\text { Diplazium matthewii(Copel.) } \\
\text { C.Chr }\end{array}$ & Nieuro & Athyriaceae & $\mathrm{H}$ & Sh & $\begin{array}{l}\text { Young shoots are } \\
\text { cooked and taken } \\
\text { in stomach } \\
\text { problem }\end{array}$ & $\begin{array}{l}\text { Rana et al., } \\
2015\end{array}$ & 1 \\
\hline 129. & $\begin{array}{l}\text { Drymaria cordata (L.) Willd. Ex } \\
\text { Schult }\end{array}$ & Abijalo & Caryophyllaceae & Herb & $\mathrm{Wp}$ & $\begin{array}{l}\text { Plant juice is taken } \\
\text { in stomach } \\
\text { problem and } \\
\text { indigestion. Plant } \\
\text { is mixed with rice } \\
\text { flour and fried in } \\
\text { ghee making bread } \\
\text { which is used to } \\
\text { cure stomach pain. }\end{array}$ & $\begin{array}{l}\text { Adhikari et al., } \\
\text { 2019; Ambu } \text { et } \\
\text { al., 2020; Rana } \\
\text { et al., 2015; } \\
\text { Joshi } \text { et al., } \\
\text { 2011; Acharya, } \\
\text { 2012; Joshi et } \\
\text { al., 2018 }\end{array}$ & 6 \\
\hline 130. & $\begin{array}{l}\text { Duchesnea indica (Andrews) } \\
\text { Focke }\end{array}$ & Bhui kafal & Rosaceae & $\mathrm{H}$ & F, Up & $\begin{array}{l}\text { Root is used in } \\
\text { stomach problem. } \\
\text { Fruit is taken as } \\
\text { laxative. }\end{array}$ & $\begin{array}{l}\text { Rana et al., } \\
\text { 2015; Kunwar } \\
\text { \& Adhikari, } \\
2005\end{array}$ & 2 \\
\hline 131. & Elephantopus scaber L. & Sahashrabuti & Asteraceae & $\mathrm{H}$ & $\mathrm{Wp}$ & $\begin{array}{l}\text { plant extract is } \\
\text { used in } \\
\text { constipation. }\end{array}$ & Dhami, 2008 & 1 \\
\hline 132. & Ephedra gerardiana Wall. & Ephedra & Ephedraceae & $\mathrm{S}$ & $F$ & $\begin{array}{l}\text { Ripe fruits are } \\
\text { given to cure } \\
\text { indigestion. }\end{array}$ & $\begin{array}{l}\text { kunwar \& } \\
\text { Adhikari, } 2005\end{array}$ & 1 \\
\hline 133. & $\begin{array}{l}\text { Euodia fraxinifolia (D.Don) } \\
\text { Hook.f. }\end{array}$ & & Ruta & $\mathrm{T}$ & $\mathrm{F}$ & $\begin{array}{l}\text { Seed paste mixed } \\
\text { with water and } \\
\text { consumrd during } \\
\text { indigestion and } \\
\text { stomach disorders. }\end{array}$ & $\begin{array}{l}\text { Singh et al., } \\
2016\end{array}$ & 1 \\
\hline 134. & Euphorbia hirta L. & Dudhejhar & Euphorbiaceae & $\mathrm{H}$ & $\mathrm{Wp}$ & $\begin{array}{l}\text { Leaf juice is given } \\
\text { orally in diarrhoea. } \\
\text { Plant extract is } \\
\text { used to treat } \\
\text { diarrhoea and } \\
\text { dysentery. }\end{array}$ & $\begin{array}{l}\text { Singh et al., } \\
\text { 2012; Dhami, } \\
2008\end{array}$ & 2 \\
\hline 135. & Euphorbia royleana Boiss. & Sieudi & Euphorbiaceae & $\mathrm{S}$ & $\mathrm{L}, \mathrm{S}$ & $\begin{array}{l}\text { Stem is used for } \\
\text { stomach disorders } \\
\text { during indigestion } \\
\text { and } \\
\text { constipation.Leaf } \\
\text { juice is used to } \\
\text { treat diarrhoea, } \\
\text { dysentery and } \\
\text { constipation. }\end{array}$ & $\begin{array}{l}\text { Manandhar, } \\
\text { 1990; Bhattarai } \\
\text { et al., } 2009\end{array}$ & 2 \\
\hline 136. & $\begin{array}{l}\text { Fagopyrum dibotrys (D.Don) } \\
\text { H.Hara }\end{array}$ & & Polygonaceae & $\mathrm{H}$ & Up & $\begin{array}{l}\text { Dry root powder is } \\
\text { consumed to treat } \\
\text { diarrhoea and } \\
\text { dysentery. }\end{array}$ & $\begin{array}{l}\text { Rokaya et al., } \\
2010\end{array}$ & 1 \\
\hline 137. & $\begin{array}{l}\text { Fagopyrum esculentum } \\
\text { (Moench) }\end{array}$ & Mithephapar & Polygonaceae & $\mathrm{H}$ & $\mathrm{F}$ & $\begin{array}{l}\text { Seed flour is used } \\
\text { to make cakes } \\
\text { which is beneficial } \\
\text { for indigestion and } \\
\text { diarrhoea. }\end{array}$ & $\begin{array}{l}\text { Rokaya et al., } \\
2010\end{array}$ & 1 \\
\hline
\end{tabular}




\begin{tabular}{|c|c|c|c|c|c|c|c|c|}
\hline 138. & $\begin{array}{l}\text { Fagopyrum tataricum (L.) } \\
\text { Gaertn. }\end{array}$ & & Polygonaceae & $\mathrm{H}$ & $\mathrm{F}$ & $\begin{array}{l}\text { Seed flour cakes } \\
\text { are taken in } \\
\text { indigestion and } \\
\text { diarrhoea. }\end{array}$ & $\begin{array}{l}\text { Rokaya et al., } \\
2010\end{array}$ & 1 \\
\hline 139. & Ficus religiosa L. & Pipal & Moraceae & $\mathrm{T}$ & $\mathrm{B}$ & $\begin{array}{l}\text { Bark juice is used } \\
\text { to treat diarrhoea } \\
\text { and dysentery. }\end{array}$ & $\begin{array}{l}\text { Dhami, 2008; } \\
\text { Joshi et al., } \\
2011\end{array}$ & 2 \\
\hline 140. & Ficus auriculata Lour. & Timilo & Moraceae & $\mathrm{T}$ & $\mathrm{F}, \mathrm{Sh}$ & $\begin{array}{l}\text { Tender shoot and } \\
\text { fruit Is used to } \\
\text { treat diarrhoea and } \\
\text { dysentery. }\end{array}$ & Dhami, 2008 & 1 \\
\hline 141. & Ficus benghalensis L. & Bar & Moraceae & $\mathrm{T}$ & $\mathrm{B}, \mathrm{L}$ & $\begin{array}{l}\text { Tender leaves are } \\
\text { used to cure } \\
\text { cholera and } \\
\text { dysentery. Bark } \\
\text { infusion is used in } \\
\text { diarrhoea and } \\
\text { dysentery. }\end{array}$ & $\begin{array}{l}\text { Acharya, 2012; } \\
\text { Acharya and } \\
\text { Acharya, 2009 }\end{array}$ & 2 \\
\hline 142. & $\begin{array}{l}\text { Ficus semicordata Buch.-Ham ex } \\
\text { Sm. }\end{array}$ & Khanayo & Moraceae & $\mathrm{T}$ & $\mathrm{B}, \mathrm{F}$ & $\begin{array}{l}\text { Fig is eaten raw to } \\
\text { treat constipation } \\
\text { and indigestion. } \\
\text { Bark extract is } \\
\text { used tomtreat } \\
\text { dysentery. }\end{array}$ & $\begin{array}{l}\text { Manandhar, } \\
\text { 1990; Dhami, } \\
\text { 2008; Ambu et } \\
\text { al., 2020. }\end{array}$ & 3 \\
\hline 143. & Garuga pinnata Roxb. & Dabdabe & Burser & $\mathrm{T}$ & $\mathrm{B}$ & $\begin{array}{l}\text { Bark is used to } \\
\text { treat stomach } \\
\text { disorders. }\end{array}$ & $\begin{array}{l}\text { Manandhar, } \\
1990\end{array}$ & 1 \\
\hline 144. & Gaultheria fragrantissima Wall. & Dhasingare & Ericaceae & $\mathrm{T}$ & $\mathrm{F}, \mathrm{L}$ & $\begin{array}{l}\text { Leaves and } \\
\text { immature fruits are } \\
\text { consumed to treat } \\
\text { gastritis. Fruit } \\
\text { paste is used to } \\
\text { treat intestinal } \\
\text { worms. }\end{array}$ & $\begin{array}{l}\text { Joshi et al., } \\
\text { 2011; Acharya, } \\
2012\end{array}$ & 2 \\
\hline 145. & $\begin{array}{l}\text { Gentiana pedicellata (D.Don) } \\
\text { Griseb. }\end{array}$ & Tukephool & Gentianaceae & $\mathrm{H}$ & $\mathrm{Wp}$ & $\begin{array}{l}\text { Plant juice is given } \\
\text { as anthelmintic. }\end{array}$ & $\begin{array}{l}\text { Manandhar, } \\
1990\end{array}$ & 1 \\
\hline 146. & $\begin{array}{l}\text { Girardinia diversifolia (Link.) } \\
\text { Friis. }\end{array}$ & Allo & Urticaceae & $\mathrm{S}$ & Up & $\begin{array}{l}\text { Root juice is taken } \\
\text { orally in gastric } \\
\text { disorders and } \\
\text { constipation. }\end{array}$ & $\begin{array}{l}\text { Rana et al., } \\
\text { 2015; Malla et } \\
\text { al., } 2015\end{array}$ & 2 \\
\hline 147. & Glycyrrhiza glabra L. & Jethimadhu & Fabaceae & $\mathrm{H}$ & Up & $\begin{array}{l}\text { Root juice is used } \\
\text { in indigestion. }\end{array}$ & $\begin{array}{l}\text { Malla et al., } \\
2015\end{array}$ & 1 \\
\hline 148. & Gonostegia hirta (B1.) Miq. & Maslahari & Urticaceae & $\mathrm{H}$ & Wp & $\begin{array}{l}\text { Plant juice is } \\
\text { mixed with juice } \\
\text { of Drymaria } \\
\text { diandra and } \\
\text { Centella asiatica to } \\
\text { treat diarrhoea }\end{array}$ & $\begin{array}{l}\text { Manandhar, } \\
1990\end{array}$ & 1 \\
\hline 149. & Grewia serrulata DC. & Syalpuchhere & Malvaceae & $\mathrm{T}$ & $\mathrm{B}$ & $\begin{array}{l}\text { Bark juice is given } \\
\text { in stomach } \\
\text { disorders. }\end{array}$ & $\begin{array}{l}\text { Manandhar, } \\
1990\end{array}$ & 1 \\
\hline 150. & Hedychium spicatum Sm. & Camera & Zingiberaceae & $\mathrm{H}$ & Up & $\begin{array}{l}\text { Rhizome decoction } \\
\text { is used in } \\
\text { indigestion. }\end{array}$ & $\begin{array}{l}\text { Malla et al., } \\
2015\end{array}$ & 1 \\
\hline 151. & Hedyotis scandens Roxb. & Bakhari lahara & Rubiaceae & $\mathrm{C}$ & $\mathrm{B}$ & $\begin{array}{l}\text { Bark juice is given } \\
\text { in stomach } \\
\text { disorders. }\end{array}$ & $\begin{array}{l}\text { Manandhar, } \\
1990\end{array}$ & 1 \\
\hline 152. & $\begin{array}{l}\text { Henckelia urticifolia (Buch.- } \\
\text { Ham. ex D.Don) A. Dietr }\end{array}$ & Aankhle jhar & Gesneriaceae & $\mathrm{H}$ & $\mathrm{Wp}$ & $\begin{array}{l}\text { Plant decoction is } \\
\text { given to } \\
\text { stomachache. }\end{array}$ & $\begin{array}{l}\text { Malla et al., } \\
2015\end{array}$ & 1 \\
\hline 153. & Heracleum nepalense D.Don. & & Apiaceae & $\mathrm{S}$ & $\mathrm{F}$ & $\begin{array}{l}\text { Seed pickles is } \\
\text { used to treat } \\
\text { stomach disorders. }\end{array}$ & $\begin{array}{l}\text { Singh et al., } \\
2016\end{array}$ & 1 \\
\hline
\end{tabular}




\begin{tabular}{|c|c|c|c|c|c|c|c|c|}
\hline 154. & Heracleum wallichii DC. & & Apiaceae & $\mathrm{H}$ & $\mathrm{F}, \mathrm{Up}$ & $\begin{array}{l}\text { Root powder is } \\
\text { taken with hot } \\
\text { water to treat } \\
\text { stomachache. Fruit } \\
\text { is used to treat } \\
\text { stomachache. }\end{array}$ & $\begin{array}{l}\text { Rokaya } \text { et al., } \\
\text { 2010; Limbu \& } \\
\text { Rai, } 2013\end{array}$ & 2 \\
\hline 155. & Heynea trijuga Roxb. ex Sims. & & Meliaceae & $\mathrm{T}$ & $\mathrm{B}, \mathrm{L}$ & $\begin{array}{l}\text { Bark and leaf } \\
\text { decoction is taken } \\
\text { orally in cholera. }\end{array}$ & $\begin{array}{l}\text { Singh et al., } \\
2018\end{array}$ & 1 \\
\hline 156. & Hippophae salicifolia D.Don & & Elaeagnaceae & $\mathrm{T}$ & $\mathrm{F}$ & $\begin{array}{l}\text { Fruit decoction is } \\
\text { used to kill } \\
\text { stomach worms. }\end{array}$ & $\begin{array}{l}\text { Rokaya et al., } \\
2010\end{array}$ & 1 \\
\hline 157. & Hippophae tibetana Schltdl.. & & Elaeagnaceae & $\mathrm{T}$ & $\mathrm{F}$ & $\begin{array}{l}\text { Fruit juice or } \\
\text { decoction is } \\
\text { consumed as } \\
\text { apetizer and } \\
\text { anthelmintic. }\end{array}$ & $\begin{array}{l}\text { Rokaya et al., } \\
2010\end{array}$ & 1 \\
\hline 158. & Hypericum cordifolium Choisy. & & Hypericaceae & $\mathrm{S}$ & Up & $\begin{array}{l}\text { Root juice is used } \\
\text { to cure diarrhoea. }\end{array}$ & $\begin{array}{l}\text { Manandhar, } \\
1990\end{array}$ & 1 \\
\hline 159. & Hypericum japonicum Thunb. & Kanikeghans & Hypericaceae & $\mathrm{H}$ & Wp & $\begin{array}{l}\text { Plant juice is used } \\
\text { in dysentery and } \\
\text { indigestion. }\end{array}$ & $\begin{array}{l}\text { Malla et al., } \\
2015\end{array}$ & 1 \\
\hline 160. & Impatiens puberula DC. & Bhendeghans & Balsaminaceae & $\mathrm{H}$ & Wp & $\begin{array}{l}\text { Whole plant is } \\
\text { recommended in } \\
\text { indigestion. }\end{array}$ & $\begin{array}{l}\text { Malla et al., } \\
2015\end{array}$ & 1 \\
\hline 161. & Imperata cylindrica (L.) Raeush. & Siru & Poaceae & $\mathrm{H}$ & $\mathrm{Up}$ & $\begin{array}{l}\text { Rhizome is used to } \\
\text { treat gastritis and } \\
\text { dysentery and } \\
\text { indigestion }\end{array}$ & $\begin{array}{l}\text { Acharya, 2012; } \\
\text { Dhami, } 2008\end{array}$ & 2 \\
\hline 162. & $\begin{array}{l}\text { Inula cappa (Buch.-Ham. ex } \\
\text { D.Don) DC }\end{array}$ & Gaitihare & Asteraceae & $\mathrm{S}$ & Up & $\begin{array}{l}\text { Root juice is taken } \\
\text { to treat gastritis } \\
\text { and indigestion. }\end{array}$ & $\begin{array}{l}\text { Joshi et al., } \\
2011 .\end{array}$ & 1 \\
\hline 163. & Ipomoea aquatica Forssk. & & Convolvulaceae & $\mathrm{H}$ & $\mathrm{Sh}$ & $\begin{array}{l}\text { Tender shoot is } \\
\text { used as vegetable } \\
\text { in gastric trouble. }\end{array}$ & $\begin{array}{l}\text { Singh et al., } \\
2012\end{array}$ & 1 \\
\hline 164. & Ipomoea purpurea (L.) Roth. & and & Convolvulaceae & $\mathrm{C}$ & $\mathrm{L}$ & $\begin{array}{l}\text { Leaf decoction is } \\
\text { used in diarrhoea. }\end{array}$ & $\begin{array}{l}\text { Malla et al., } \\
2015\end{array}$ & 1 \\
\hline 165. & $\begin{array}{l}\text { Iris domestica (L.) Goldblatt \& } \\
\text { Mabb. }\end{array}$ & & Iridaceae & $\mathrm{H}$ & Up & $\begin{array}{l}\text { Root juice is used } \\
\text { to treat gastric } \\
\text { problem. }\end{array}$ & $\begin{array}{l}\text { Ambu et al., } \\
2020\end{array}$ & 1 \\
\hline 166. & Juglans regia L. & Okhar & Juglandaceae & $\mathrm{T}$ & $\mathrm{B}$ & $\begin{array}{l}\text { Bark is used to } \\
\text { treat intestinal } \\
\text { worms. }\end{array}$ & $\begin{array}{l}\text { kunwar \& } \\
\text { Adhikari, 2005; } \\
\text { Acharya, 2012 }\end{array}$ & 2 \\
\hline 167. & $\begin{array}{l}\text { Juniperus recurva Buch.-Ham ex } \\
\text { D.Don }\end{array}$ & & Cupressaceae & $\mathrm{S}$ & $\mathrm{F}, \mathrm{L}$ & $\begin{array}{l}\text { Leaves and fruit } \\
\text { powder is taken } \\
\text { with hot water to } \\
\text { treat diarrhoea. }\end{array}$ & $\begin{array}{l}\text { Rokaya et al., } \\
2010\end{array}$ & 1 \\
\hline 168. & Jurinea dolomiaea Boiss & & Asteraceae & $\mathrm{H}$ & $\mathrm{Wp}$ & $\begin{array}{l}\text { Whole plant } \\
\text { powder is taken } \\
\text { with hot water to } \\
\text { treat diarrhoea, } \\
\text { dysentery and } \\
\text { stomachache. }\end{array}$ & $\begin{array}{l}\text { Rokaya et al., } \\
\text { 2010; Kunwar } \\
\text { \& Adhikari, } \\
2005\end{array}$ & 12 \\
\hline 169. & Lablab purpureus (L.) Sweet & Tatesimi & Fabaceae & $\mathrm{C}$ & $\mathrm{L}$ & $\begin{array}{l}\text { Leaf juice is used } \\
\text { to treat } \\
\text { constipation. }\end{array}$ & $\begin{array}{l}\text { Ambu et al., } \\
2020\end{array}$ & 1 \\
\hline 170. & $\begin{array}{l}\text { Lagenaria siceraria } \\
\text { (Molina)Standl. }\end{array}$ & Lauka & Cucurbitaceae & $\mathrm{C}$ & $\mathrm{F}$ & $\begin{array}{l}\text { Fruit juice is given } \\
\text { in diarrhoea, } \\
\text { dysentery. }\end{array}$ & $\begin{array}{l}\text { Singh et al., } \\
2012\end{array}$ & 1 \\
\hline
\end{tabular}




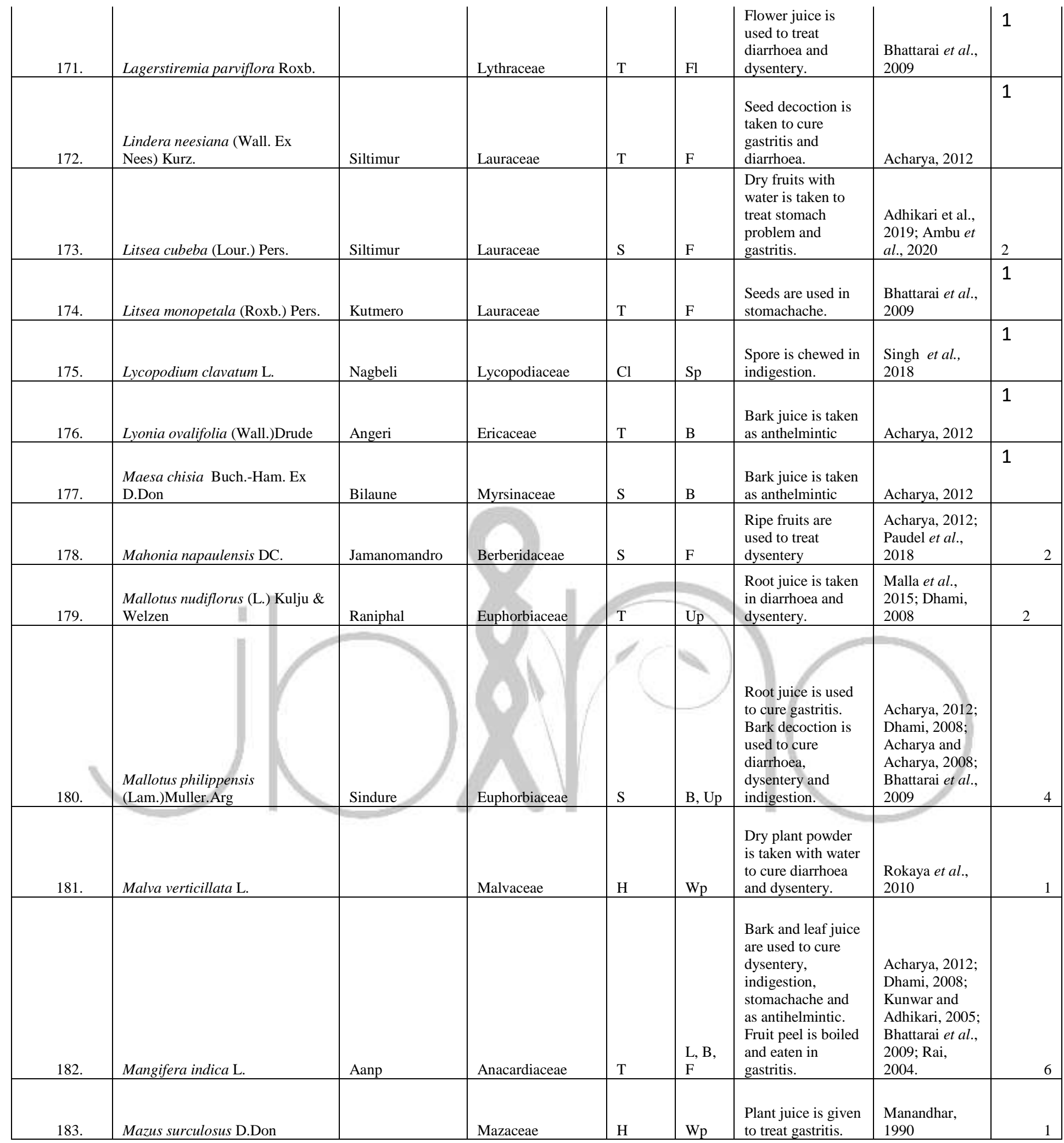




\begin{tabular}{|c|c|c|c|c|c|c|c|c|}
\hline 184. & Melia azadirchta L. & Bakaino & Meliaceae & $\mathrm{T}$ & $\mathrm{B}, \mathrm{F}$ & $\begin{array}{l}\text { Bark juice and } \\
\text { fruit is used as } \\
\text { anthelmintic and } \\
\text { used to treat } \\
\text { diarrhoea and } \\
\text { gastric infection. }\end{array}$ & $\begin{array}{l}\text { Acharya, 2012; } \\
\text { Dhami, 2008; } \\
\text { Acharya and } \\
\text { Acharya, 2009; } \\
\text { Ambu et al., } \\
\text { 2020; Bhattarai } \\
\text { et al., } 2009\end{array}$ & 6 \\
\hline 185. & Mentha arvensis L. & & Lamiaceae & $\mathrm{H}$ & $\mathrm{L}$ & $\begin{array}{l}\text { Leaf juice is used } \\
\text { to treat gastritis } \\
\text { and stomachache. }\end{array}$ & $\begin{array}{l}\text { Bhattarai et al., } \\
\text { 2009; Paudel et } \\
\text { al., } 2018\end{array}$ & 2 \\
\hline 186. & Mentha spicata $\mathrm{L}$. & Babari & Lamiaceae & $\mathrm{H}$ & Wp & $\begin{array}{l}\text { Whole plant juice } \\
\text { is orally taken in } \\
\text { diarrhoea and } \\
\text { stomachache. Leaf } \\
\text { is used in gastritis, } \\
\text { diarrhoea and } \\
\text { dysentery. }\end{array}$ & $\begin{array}{l}\text { Adhikari et al., } \\
\text { 2019; Rokaya } \\
\text { et al., 2010; } \\
\text { Acharya, 2012; } \\
\text { Acharya and } \\
\text { Acharya, } 2009\end{array}$ & 4 \\
\hline 187. & Mesua ferrea $\mathrm{L}$. & & Calophyllaceae & $\mathrm{T}$ & $\mathrm{Fl}$ & $\begin{array}{l}\text { Flower paste is } \\
\text { given in amoebic } \\
\text { dysentery. }\end{array}$ & $\begin{array}{l}\text { Manandhar, } \\
1990\end{array}$ & 1 \\
\hline 188. & Michelia champaca L. & Champ & Magnoliaceae & $\mathrm{T}$ & $\mathrm{B}$ & $\begin{array}{l}\text { Bark juice is used } \\
\text { to treat gastritis. }\end{array}$ & Acharya, 2012 & 1 \\
\hline 189. & Mimosa pudica L. & Lajjabati jhar & Fabaceae & $\mathrm{H}$ & & $\begin{array}{l}\text { Plant decoction is } \\
\text { given in diarrhoea } \\
\text { and dysentery. } \\
\text { Root juice is taken } \\
\text { to treat } \\
\text { stomachache. }\end{array}$ & $\begin{array}{l}\text { Singh et al., } \\
\text { 2012; Joshi et } \\
\text { al., 2018; } \\
\text { Manandhar, } \\
1987\end{array}$ & 3 \\
\hline 190. & Mirabilis jalapa L. & Lankajalapa & Nyctaginaceae & $\mathrm{S}$ & Up & $\begin{array}{l}\text { Root paste is used } \\
\text { to treat diarrhoea. }\end{array}$ & $\begin{array}{l}\text { Manandhar, } \\
1987\end{array}$ & 1 \\
\hline 191. & Morus indica L. & Kimbu & Moraceae & $\mathrm{T}$ & $\mathrm{F}$ & $\begin{array}{l}\text { Fruit is eaten raw } \\
\text { in diarrhoea and } \\
\text { dysentery. }\end{array}$ & $\begin{array}{l}\text { Singh et al., } \\
2018\end{array}$ & 1 \\
\hline 192. & Morus serrata Roxb. & Kimbu & Moraceae & $\mathrm{T}$ & F, Up & $\begin{array}{l}\text { Fruit is beneficial } \\
\text { in diarrhoea, } \\
\text { indigestion and } \\
\text { constipation. Root } \\
\text { juice is used as } \\
\text { antihelmintic. Root } \\
\text { paste with water is } \\
\text { used to treat } \\
\text { diarrhoea. }\end{array}$ & $\begin{array}{l}\text { Rokaya et al., } \\
\text { 2010; Dhami, } \\
\text { 2008; Bhattarai } \\
\text { et al., } 2009\end{array}$ & 3 \\
\hline 193. & Murraya koenigii (L.) Spreng & Karripatta & Rutaceae & $\mathrm{T}$ & $\mathrm{B}, \mathrm{L}$ & $\begin{array}{l}\text { Bark and leaf juice } \\
\text { is used to treat } \\
\text { stomachache, } \\
\text { diarrhoea and } \\
\text { dysentery. }\end{array}$ & $\begin{array}{l}\text { Dhami, 2008; } \\
\text { Acharya and } \\
\text { Acharya, 2009; } \\
\text { Joshi et al., } \\
\text { 2018; Paudel et } \\
\text { al., 2018 }\end{array}$ & 4 \\
\hline 194. & Musa paradiisiaca $\mathrm{L}$. & Kera & Musaceae & $\mathrm{S}$ & $\mathrm{F}$ & $\begin{array}{l}\text { Unripe fruits are } \\
\text { given orally in } \\
\text { diarrhoea and } \\
\text { dysentery. }\end{array}$ & $\begin{array}{l}\text { Singh et al., } \\
\text { 2012; Dhami, } \\
2008\end{array}$ & 2 \\
\hline
\end{tabular}




\begin{tabular}{|c|c|c|c|c|c|c|c|c|}
\hline 195. & $\begin{array}{l}\text { Myrica esculenta Buch.-Ham. ex } \\
\text { D.Don }\end{array}$ & Kaphal & Myricaceae & $\mathrm{T}$ & $\mathrm{B}$ & $\begin{array}{l}\text { Bark juice is given } \\
\text { to treat stomach } \\
\text { disorders and } \\
\text { diarrhoea. }\end{array}$ & $\begin{array}{l}\text { Manandhar, } \\
\text { 1990; Shrestha } \\
\text { \& Dhillon, } \\
2003\end{array}$ & 2 \\
\hline 196. & Nardostachys grandiflora & Jatamansi & Valerianaceae & $\mathrm{H}$ & Up & $\begin{array}{l}\text { Rhizome infusion } \\
\text { is taken in stomatal } \\
\text { disorders and } \\
\text { intestinal worms. }\end{array}$ & $\begin{array}{l}\text { Rokaya et al., } \\
2010\end{array}$ & 1 \\
\hline 197. & $\begin{array}{l}\text { Neopicrorhiza scrophulariiflora } \\
\text { (Pennell) Hong }\end{array}$ & Kutki & Scrophulariaceae & $\mathrm{H}$ & Up & $\begin{array}{l}\text { Rhizome powder } \\
\text { is taken with hot } \\
\text { water to cure } \\
\text { stomachache. }\end{array}$ & $\begin{array}{l}\text { Rokaya et al., } \\
2010\end{array}$ & 1 \\
\hline 198. & $\begin{array}{l}\text { Nephrolepis cordifolia (L.) C. } \\
\text { Presl. }\end{array}$ & Paniamala & Nephrolepidaceae & $\mathrm{H}$ & Up & $\begin{array}{l}\text { tuber juice is used } \\
\text { in indigestion. }\end{array}$ & $\begin{array}{l}\text { Singh } \text { et } \\
\text { al.,2018; } \\
\text { Dhami, } 2008\end{array}$ & 2 \\
\hline 199. & Ocimum tenuiflorum L. & Tulsi & Lamiaceae & $\mathrm{H}$ & $\mathrm{Wp}$ & $\begin{array}{l}\text { Leaf and plant } \\
\text { juice is used to } \\
\text { treat gastric } \\
\text { problem, diarrhoea } \\
\text { and dysentery. }\end{array}$ & $\begin{array}{l}\text { Dhami, 2008; } \\
\text { Ambu et al., } \\
2020 \text {. }\end{array}$ & 2 \\
\hline 200. & Origanum vulgare L. & Ramtulsi & Lamia & $\mathrm{H}$ & $\mathrm{Wp}$ & $\begin{array}{l}\text { Dry or fresh plant } \\
\text { is boiled with } \\
\text { water and is given } \\
\text { in diarrhoea, } \\
\text { dysentery, } \\
\text { constipation and } \\
\text { stomachache. }\end{array}$ & $\begin{array}{l}\text { Rokaya et al., } \\
2010\end{array}$ & 1 \\
\hline 201. & Oroxylum indicum (L.) Kurz & Totala & Bignoniaceae & $\mathrm{T}$ & $\mathrm{F}$ & $\begin{array}{l}\text { Fruit extract is } \\
\text { used to treat } \\
\text { diarrhoea. }\end{array}$ & $\begin{array}{l}\text { Limbu \& Rai, } \\
2013\end{array}$ & 1 \\
\hline 202. & Osbeckia nutans Wall. & Sanoangeri & Melastomataceae & $\mathrm{S}$ & $\mathrm{Up}$ & $\begin{array}{l}\text { Root juice is taken } \\
\text { to treat stomach } \\
\text { disorders. }\end{array}$ & $\begin{array}{l}\text { Manandhar, } \\
1990\end{array}$ & 1 \\
\hline 203. & Oxalis corniculata L. & Chariamilo & Oxlidaceae & $\mathrm{H}$ & $\mathrm{Wp}$ & $\begin{array}{l}\text { Plant juice is given } \\
\text { to cure diarrhoea, } \\
\text { dysentery and } \\
\text { stomachache. }\end{array}$ & $\begin{array}{l}\text { Adhikari et al., } \\
\text { 2019; Rokaya } \\
\text { et al., 2010; } \\
\text { Joshi et al., } \\
\text { 2011; Acharya, } \\
\text { 2012; Dhami, } \\
\text { 2008; Acharya } \\
\text { \& Acharya, } \\
2009\end{array}$ & 6 \\
\hline 204. & Oxalis debilis Kunth. & Chariamilo & Oxlidaceae & $\mathrm{H}$ & $\mathrm{Wp}$ & $\begin{array}{l}\text { Plant juice is given } \\
\text { in the treatment of } \\
\text { acidity, diarrhoea } \\
\text { and dysentery. }\end{array}$ & $\begin{array}{l}\text { Malla et al., } \\
2015\end{array}$ & 1 \\
\hline 205. & Paris polyphylla $\mathrm{Sm}$. & Satuwa & Liliaceae & $\mathrm{H}$ & Up & $\begin{array}{l}\text { Rhizome juice is } \\
\text { given in gastritis. }\end{array}$ & $\begin{array}{l}\text { Malla et al., } \\
2015\end{array}$ & 1 \\
\hline 206. & $\begin{array}{l}\text { Parnassia wightiana Wall. Ex } \\
\text { Wight \& Arn. }\end{array}$ & Sunakhari & Celastraceae & $\mathrm{H}$ & $\mathrm{L}$ & $\begin{array}{l}\text { Leaf juice is given } \\
\text { for constipation } \\
\text { and ulcers. }\end{array}$ & $\begin{array}{l}\text { Malla et al., } \\
2015\end{array}$ & 1 \\
\hline 207. & $\begin{array}{l}\text { Pedicularis gracilis Wall. Ex } \\
\text { Benth. }\end{array}$ & & Scrophulariaceae & $\mathrm{H}$ & Up & $\begin{array}{l}\text { Root extract is } \\
\text { given in } \\
\text { stomachache. }\end{array}$ & $\begin{array}{l}\text { Malla et al., } \\
2015\end{array}$ & 1 \\
\hline 208. & $\begin{array}{l}\text { Persicaria capitata (Buch.- Ham. } \\
\text { Ex D.Don)H.Gross }\end{array}$ & Pirre & Polygonaceae & $\mathrm{H}$ & $\mathrm{Wp}$ & $\begin{array}{l}\text { plant juice is given } \\
\text { in stomachache. }\end{array}$ & $\begin{array}{l}\text { Manandhar, } \\
1987\end{array}$ & 1 \\
\hline
\end{tabular}




\begin{tabular}{|c|c|c|c|c|c|c|c|c|}
\hline 209. & $\begin{array}{l}\text { Phyllanthus amarus Schumach \& } \\
\text { Thonn }\end{array}$ & Bhuiamala & Phyllanthaceae & $\mathrm{H}$ & $\mathrm{L}$ & $\begin{array}{l}\text { Leaves extract is } \\
\text { given in diarrhoea. }\end{array}$ & $\begin{array}{l}\text { Singh et al., } \\
2018\end{array}$ & 1 \\
\hline 210. & Phyllanthus emblica $\mathrm{L}$. & Amala & Phyllanthaceae & $\mathrm{T}$ & $\begin{array}{l}\mathrm{B}, \mathrm{F} \\
\mathrm{L}\end{array}$ & $\begin{array}{l}\text { Bark and leaf juice } \\
\text { is given in } \\
\text { diarrhoea,dysenter } \\
\text { y and constipation. } \\
\text { Fruit is useful in } \\
\text { stomachache. }\end{array}$ & $\begin{array}{l}\text { Singh et al., } \\
\text { 2012; Dhami, } \\
\text { 2008; } \\
\text { Manandhar, } \\
\text { 1990; Joshi et } \\
\text { al., 2018 }\end{array}$ & 4 \\
\hline 211. & Phytolacca acinosa Roxb. & & Phytolaccaceae & $\mathrm{H}$ & $\mathrm{Up}$ & $\begin{array}{l}\text { Root juice is used } \\
\text { to treat } \\
\text { constipation. }\end{array}$ & $\begin{array}{l}\text { Shrestha \& } \\
\text { Dhillon, } 2003\end{array}$ & 1 \\
\hline 212. & Pinus roxburghii Sargent & Sallo & Pinaceae & $\mathrm{T}$ & $\operatorname{Re}$ & $\begin{array}{l}\text { Resin is used in } \\
\text { stomachache. }\end{array}$ & Acharya, 2012 & 1 \\
\hline 213. & Piper longum $\mathrm{L}$. & Pipla & Piperaceae & $\mathrm{H}$ & $\begin{array}{l}\text { F, L, } \\
\text { Up }\end{array}$ & $\begin{array}{l}\text { Fruit powder is } \\
\text { given in } \\
\text { constipation and } \\
\text { indigestion. Leaf } \\
\text { powder infusion is } \\
\text { used to treat } \\
\text { stomachache.Root } \\
\text { infusion is used to } \\
\text { treat stomachache. }\end{array}$ & $\begin{array}{l}\text { Malla et } \\
\text { al.,2015; } \\
\text { Acharya, 2012; } \\
\text { Joshi et al., } \\
2018\end{array}$ & 3 \\
\hline 214. & Piper retrofractum Vahl. & & Piperaceae & $\mathrm{C}$ & $\mathrm{S}$ & $\begin{array}{l}\text { Stem is used to } \\
\text { treat gastric } \\
\text { disorders. }\end{array}$ & $\begin{array}{l}\text { Ambu et al., } \\
2020\end{array}$ & 1 \\
\hline 215. & Pisidium guajava $\mathrm{L}$. & Amba & Myrtaceae & $\mathrm{T}$ & $\begin{array}{l}\text { B, } \\
\text { Bud }\end{array}$ & $\begin{array}{l}\text { Young buds } \\
\text { decoctionand juice } \\
\text { is used in the } \\
\text { treatment of } \\
\text { diarrhoea and } \\
\text { dysentery. Bark } \\
\text { extract is used to } \\
\text { treat diarrhoea and } \\
\text { relieve from blood } \\
\text { in stool. }\end{array}$ & $\begin{array}{l}\text { Dhami, } 2008 . \\
\text { Limbu \& Rai, } \\
\text { 2013. Ambu et } \\
\text { al., 2020. } \\
\text { Bhattarai et al., } \\
2009\end{array}$ & 4 \\
\hline 216. & Plantago major L. & 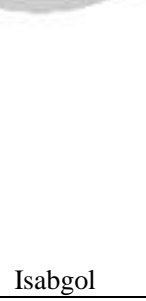 & Plantaginaceae & $\mathrm{H}$ & $\mathrm{Wp}$ & $\begin{array}{l}\text { Seeds are used to } \\
\text { cure indigestion, } \\
\text { dysentery and } \\
\text { diarrhoea. Dry } \\
\text { plant powder is } \\
\text { taken with water to } \\
\text { cure diarrhoea and } \\
\text { dysentery. }\end{array}$ & $\begin{array}{l}\text { 2019; Joshi et } \\
\text { al., 2011; } \\
\text { Acharya, 2012; } \\
\text { Acharya \& } \\
\text { Acharya, 2009; } \\
\text { Kunwar \& } \\
\text { Adhikari, 2005; } \\
\text { Rokaya et al, } \\
2010\end{array}$ & 6 \\
\hline 217. & $\begin{array}{l}\text { Pleurospermum dentatum (DC.) } \\
\text { C.B Clarke }\end{array}$ & & Apiaceae & $\mathrm{H}$ & $\mathrm{Up}$ & $\begin{array}{l}\text { Root powder is } \\
\text { taken orally for } \\
\text { indigestion. }\end{array}$ & $\begin{array}{l}\text { Rokaya et al., } \\
2010\end{array}$ & 1 \\
\hline 218. & Plumbago zeylanica $\mathrm{L}$. & & Plumbaginaceae & $\mathrm{H}$ & $\mathrm{Wp}$ & $\begin{array}{l}\text { Plant juice is used } \\
\text { to treat gastric } \\
\text { disorders. }\end{array}$ & $\begin{array}{l}\text { Ambu et al., } \\
2020\end{array}$ & 1 \\
\hline 219. & Pogostemon glaber Benth. & Rudilo & Lamiaceae & $\mathrm{S}$ & Up & $\begin{array}{l}\text { Root juice is given } \\
\text { in indigestion. }\end{array}$ & $\begin{array}{l}\text { Malla et } \\
\text { al.,2015 }\end{array}$ & 1 \\
\hline 220. & $\begin{array}{l}\text { Polygonatum cirrhifolium } \\
\text { (Wall.) Royle }\end{array}$ & Khiraulo & Liliaceae & $\mathrm{H}$ & Up & $\begin{array}{l}\text { Root juice is orally } \\
\text { given to treat } \\
\text { diarrhoea. }\end{array}$ & $\begin{array}{l}\text { Rokaya et al., } \\
2010\end{array}$ & 1 \\
\hline
\end{tabular}




\begin{tabular}{|c|c|c|c|c|c|c|c|c|}
\hline 221. & Polygonum plebeium $\mathrm{R} . \mathrm{Br}$ & Balaunisag & Polygonaceae & $\mathrm{H}$ & $\mathrm{Wp}$ & $\begin{array}{l}\text { Plant boiled with } \\
\text { some water and } \\
\text { goven in bllody } \\
\text { dysentery }\end{array}$ & $\begin{array}{l}\text { Manandhar, } \\
1990\end{array}$ & 1 \\
\hline 222. & Potentilla microphylla D.Don & Bajradandti & Rosaceae & $\mathrm{H}$ & $\mathrm{L}, \mathrm{Up}$ & $\begin{array}{l}\text { Root paste is taken } \\
\text { in diarrhoea. Leaf } \\
\text { juice is given in } \\
\text { dysentery. }\end{array}$ & $\begin{array}{l}\text { kunwar \& } \\
\text { Adhikari, 2005; } \\
\text { Acharya, 2012 }\end{array}$ & 2 \\
\hline 223. & Prunus armeniaca L. & & Rosaceae & $\mathrm{T}$ & $\mathrm{Re}$ & $\begin{array}{l}\text { Resin is taken } \\
\text { orally to treat } \\
\text { diarrhoea. }\end{array}$ & $\begin{array}{l}\text { Rokaya et al., } \\
2010\end{array}$ & 1 \\
\hline 224. & Prunus cerasoides D.Don & Paiyun & Rosaceae & $\mathrm{T}$ & $\mathrm{B}, \mathrm{F}$ & $\begin{array}{l}\text { Fruit is used to } \\
\text { cure gastritis and } \\
\text { bark juice is } \\
\text { antihelmintic. }\end{array}$ & Acharya, 2012 & 1 \\
\hline 225. & Pteridium aquilinum (L.) Kuhn. & Uneu & Dennstaedtiaceae & $\mathrm{H}$ & Up & $\begin{array}{l}\text { Rhizome decoction } \\
\text { is used in gastritis. }\end{array}$ & $\begin{array}{l}\text { Joshi et al., } \\
2011 .\end{array}$ & 1 \\
\hline 226. & Pterocarpus marsupium Roxb. & Bijaya sal & Fabaceae & $\mathrm{T}$ & $\mathrm{B}, \mathrm{S}$ & $\begin{array}{l}\text { Bark and wood } \\
\text { infusion is used in } \\
\text { gastritis and } \\
\text { stomachache. }\end{array}$ & Dhami, 2008 & 1 \\
\hline 227. & Punica granatum L. & Darim & Punicaceae & $\mathrm{S}$ & $\mathrm{B}, \mathrm{F}$ & $\begin{array}{l}\text { Fruit pulp, seeds } \\
\text { and bark extract } \\
\text { are eaten to treat } \\
\text { indigestion, } \\
\text { dysentery and } \\
\text { diarrhoea. }\end{array}$ & $\begin{array}{l}\text { Rokaya et al., } \\
\text { 2010; Kunwar } \\
\text { \& Adhikari, } \\
\text { 2005; Bhattarai } \\
\text { et al., } 2009\end{array}$ & 3 \\
\hline 228. & $\begin{array}{l}\text { Pyracantha crenulata (D.Don)M. } \\
\text { Roem }\end{array}$ & Ghangaru & Rosaceae & $\mathrm{S}$ & $\mathrm{F}$ & $\begin{array}{l}\text { Fruit powder withh } \\
\text { yogurt is given in } \\
\text { dysentery }\end{array}$ & $\begin{array}{l}\text { Malla et al., } \\
2015\end{array}$ & 1 \\
\hline 229. & $\begin{array}{l}\text { pyrus pashia Buch.-Ham. Ex } \\
\text { D.Don }\end{array}$ & Mayal & Rosaceae & $\mathrm{T}$ & $\mathrm{F}$ & $\begin{array}{l}\text { Fruit juice is taken } \\
\text { to cure dysentery } \\
\text { and indigestion. }\end{array}$ & $\begin{array}{l}\text { Joshi et al., } \\
\text { 2011; Acharya, } \\
\text { 2012; Shrestha } \\
\text { \& Dhillon, } \\
2003\end{array}$ & 3 \\
\hline 230. & Quercus lantana $\mathrm{Sm}$. & Banjh & Fagaceae & $\mathrm{T}$ & $\mathrm{B}$ & $\begin{array}{l}\text { Bark juice is taken } \\
\text { by the women to } \\
\text { cure gastritis after } \\
\text { delivery. }\end{array}$ & $\begin{array}{l}\text { Joshi et al., } \\
2011 \text {. }\end{array}$ & 1 \\
\hline 231. & $\begin{array}{l}\text { Ranunculus laetus Wall. Ex D. } \\
\text { Don. }\end{array}$ & & Ranunculaceae & $\mathrm{H}$ & $\mathrm{L}$ & $\begin{array}{l}\text { Leafdecoction is } \\
\text { given in } \\
\text { indigestion. }\end{array}$ & $\begin{array}{l}\text { Malla et al., } \\
2015\end{array}$ & 1 \\
\hline 232. & $\begin{array}{l}\text { Rauvolfia serpentina (L.) Benth. } \\
\text { Ex Kurz. }\end{array}$ & Sarpagandha & Apocynaceae & $\mathrm{H}$ & $\mathrm{F}$ & $\begin{array}{l}\text { Fruit is used to } \\
\text { treat Dysentery. }\end{array}$ & $\begin{array}{l}\text { Acharya and } \\
\text { Acharya, } 2009\end{array}$ & 1 \\
\hline 233. & Rhamnus virgatus Roxb. & Bhalukada & Rhamnaceae & $\mathrm{T}$ & $\mathrm{B}, \mathrm{F}$ & $\begin{array}{l}\text { Fruit and bark } \\
\text { powder is taken } \\
\text { orally in diarrhoea } \\
\text { and dysentery. }\end{array}$ & $\begin{array}{l}\text { Rokaya et al., } \\
2010\end{array}$ & 1 \\
\hline 234. & $\begin{array}{l}\text { Rheum acuminatum Hook. f. and } \\
\text { Thomson ex Hook }\end{array}$ & Padamchal & Polygonaceae & $\mathrm{H}$ & Up & $\begin{array}{l}\text { Roots or rhizomes } \\
\text { are crushed boiled } \\
\text { and consumed for } \\
\text { indigestion and } \\
\text { gastritis. Petiols } \\
\text { are also consumed } \\
\text { in diarrhoea and } \\
\text { constipation. }\end{array}$ & $\begin{array}{l}\text { Rokaya et al., } \\
\text { 2010; Singh et } \\
\text { al., 2016; } \\
\text { Shrestha \& } \\
\text { Dhillon, } 2003\end{array}$ & \\
\hline
\end{tabular}




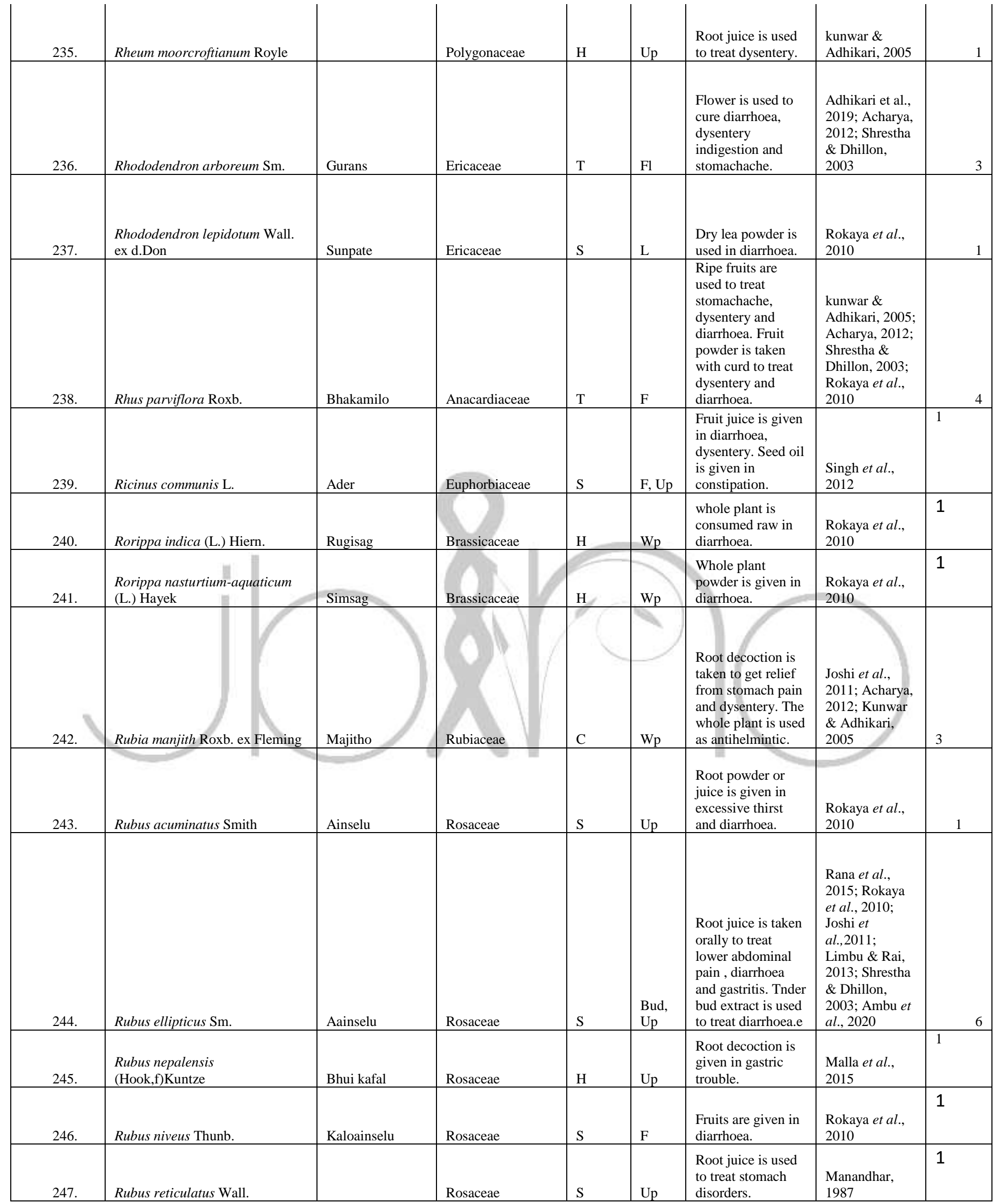




\begin{tabular}{|c|c|c|c|c|c|c|c|c|}
\hline 248. & Rumex hastatus d.Don & Raktebuj & Polygonaceae & $\mathrm{H}$ & Up & $\begin{array}{l}\text { Root powder or } \\
\text { paste is given in } \\
\text { diarrhoea and } \\
\text { dysentery. }\end{array}$ & $\begin{array}{l}\text { Rokaya } \text { et al., } \\
2010\end{array}$ & 1 \\
\hline 249. & Rumex nepalensis spreng. & Halhale & Polygonaceae & $\mathrm{H}$ & Up & $\begin{array}{l}\text { Root powder is } \\
\text { given with hot } \\
\text { water to treat } \\
\text { constipation and } \\
\text { diarrhoea. }\end{array}$ & $\begin{array}{l}\text { Adhikari et al., } \\
\text { 2019; Rokaya } \\
\text { et al., } 2010\end{array}$ & 2 \\
\hline 250. & Schima wallichii Choisy. & Chilaune & Thecaceae & $\mathrm{T}$ & $\mathrm{B}$ & $\begin{array}{l}\text { Bark powder is } \\
\text { taken with water } \\
\text { for the treatment of } \\
\text { gastritis. }\end{array}$ & $\begin{array}{l}\text { Joshi et al., } \\
2011 .\end{array}$ & 1 \\
\hline 251. & $\begin{array}{l}\text { Selinum wallichianum (DC.) } \\
\text { Raizada \& H.O. Saxena }\end{array}$ & & Apiaceae & $\mathrm{H}$ & Wp & $\begin{array}{l}\text { Root decoctionis } \\
\text { used to treat } \\
\text { diarrhoea and } \\
\text { dysentery. Plant } \\
\text { infusion is given to } \\
\text { cure stomachache } \\
\text { and diarrhoea. }\end{array}$ & $\begin{array}{l} \\
\text { kunwar \& } \\
\text { Adhikari, 2005; } \\
\text { Rokaya et al., } \\
2010 .\end{array}$ & 1 \\
\hline 252. & Semecarpus anacardium L.f. & Bhalayo & Anacardiaceae & $\mathrm{T}$ & $\mathrm{F}$ & $\begin{array}{l}\text { Fruit is used to } \\
\text { cure dysentery. }\end{array}$ & $\begin{array}{l}\text { Acharya, 2012; } \\
\text { Acharya and } \\
\text { Acharya, } 2009\end{array}$ & 2 \\
\hline 253. & $\begin{array}{l}\text { Senecio scanden Buch.-Ham. Ex } \\
\text { D.Don }\end{array}$ & Bakhrekane & Asteraceae & $\mathrm{C}$ & $\mathrm{L}$ & $\begin{array}{l}\text { Leaf juice is given } \\
\text { for gastritis. }\end{array}$ & $\begin{array}{l}\text { Malla et al., } \\
2015\end{array}$ & 1 \\
\hline 254. & Shorea robusta C.F.Gaertn. & Sal & Dipterocarpaceae & $\mathrm{T}$ & $\mathrm{Up}$ & dysentery. & et al., 2009 & 4 \\
\hline 255. & Smilex asper L. & & Liliaceae & $\mathrm{C}$ & Up & $\begin{array}{l}\text { Root juice is used } \\
\text { to cure } \\
\text { stomachache. }\end{array}$ & Acharya, 2012 & 1 \\
\hline 256. & Solanum nigrum L. & Kaligedi & Solanaceae & $\mathrm{H}$ & $\mathrm{F}$ & $\begin{array}{l}\text { Ripe fruits are } \\
\text { used to treat } \\
\text { constipation. }\end{array}$ & Dhami, 2008 & 1 \\
\hline 257. & Sonchus wightianus DC. & Mulapate & Asteraceae & $\mathrm{H}$ & Up & $\begin{array}{l}\text { Root is directly } \\
\text { taken to tret } \\
\text { gastritis. }\end{array}$ & $\begin{array}{l}\text { Singh et al. } \\
2016\end{array}$ & 1 \\
\hline 258. & Sphaeranthus senegalensis DC. & & Asteraceae & $\mathrm{H}$ & Wp & $\begin{array}{l}\text { Plant juice is used } \\
\text { to treat indigestion. }\end{array}$ & $\begin{array}{l}\text { Manandhar, } \\
1990\end{array}$ & 1 \\
\hline 259. & Stephania glandulifera Miers. & Gundarigano & Menispermaceae & & Up & $\begin{array}{l}\text { Root juice is drunk } \\
\text { to treat gastritis. }\end{array}$ & $\begin{array}{l}\text { Ambu et al., } \\
2020\end{array}$ & 1 \\
\hline 260. & $\begin{array}{l}\text { Stephania japonica (Thunb.) } \\
\text { Mier }\end{array}$ & Batulopate & Menispermaceae & $\mathrm{C}$ & $\mathrm{Up}$ & $\begin{array}{l}\text { Root juice is used } \\
\text { to treat } \\
\text { stomachache. }\end{array}$ & $\begin{array}{l}\text { Manandhar, } \\
1987\end{array}$ & 1 \\
\hline 261. & $\begin{array}{l}\text { Swertia chirayita (Roxb. Ex } \\
\text { Fleming) Karstern }\end{array}$ & Chiraita & Gentianaceae & $\mathrm{H}$ & $\mathrm{L}$ & $\begin{array}{l}\text { Leaves are used to } \\
\text { cure stomach } \\
\text { disorders, } \\
\text { indigestion and } \\
\text { constipation. }\end{array}$ & $\begin{array}{l}\text { Adhikari et al., } \\
\text { 2019; Joshi et } \\
\text { al., 2011; Joshi } \\
\text { et al., 2018; } \\
\text { Shrestha \& } \\
\text { Dhillon, } 2003\end{array}$ & 4 \\
\hline
\end{tabular}




\begin{tabular}{|c|c|c|c|c|c|c|c|c|}
\hline 262. & Syzygium cumini (L.) Skeels & Jamun & Myrtaceae & $\mathrm{T}$ & $\mathrm{B}, \mathrm{F}$ & $\begin{array}{l}\text { Bark juice is used } \\
\text { to treat diarrhoea } \\
\text { and dysentery. } \\
\text { Fruits are given to } \\
\text { cure indigstion and } \\
\text { constipation. }\end{array}$ & $\begin{array}{l}\text { Acharya, 2012; } \\
\text { Dhami, 2008; } \\
\text { L imbu \& Rai, } \\
\text { 2013; Bhattarai } \\
\text { et al., 2009; } \\
\text { Rai, 2004 }\end{array}$ & 6 \\
\hline 263. & Tamarindus indica $\mathrm{L}$. & Imili & Fabaceae & $\mathrm{T}$ & $\mathrm{F}$ & $\begin{array}{l}\text { Seed paste is used } \\
\text { to treat } \\
\text { stomachache. }\end{array}$ & Rai, 2004 & 1 \\
\hline 264. & Taraxacum officinalae Wigg. & Dudhejhar & Asteraceae & $\mathrm{H}$ & $\mathrm{Up}$ & $\begin{array}{l}\text { Root juice is given } \\
\text { to treat stomach } \\
\text { disorders. }\end{array}$ & $\begin{array}{l}\text { Manandhar, } \\
1990\end{array}$ & 1 \\
\hline 265. & $\begin{array}{l}\text { Tectaria coadunata (Wall. Ex J. } \\
\text { Sm. }\end{array}$ & Kaloneuro & Tectariaceae & $\mathrm{H}$ & Up & $\begin{array}{l}\text { Rhizome juice } \\
\text { decoction is taken } \\
\text { to treat diarrhoea, } \\
\text { dysentery, stomach } \\
\text { pain and giadriasis. }\end{array}$ & $\begin{array}{l}\text { Adhikari et al., } \\
\text { 2019; Joshi et } \\
\text { al., 2011; } \\
\text { Manandhar, } \\
\text { 1990; Limbu \& } \\
\text { Rai, 2013 }\end{array}$ & 4 \\
\hline 266. & Terminalia alata Heyne ex Roth. & Asna & Combretaceae & $\mathrm{T}$ & $\mathrm{B}$ & $\begin{array}{l}\text { Bark juice is used } \\
\text { to cure diarrhoea } \\
\text { and dysentery. }\end{array}$ & $\begin{array}{l}\text { Acharya and } \\
\text { Acharya, 2009; } \\
\text { Bhattarai et al., } \\
2009\end{array}$ & 2 \\
\hline 267. & $\begin{array}{l}\text { Terminalia bellirica (Gaertn.) } \\
\text { Roxb. }\end{array}$ & Barro & Combretaceae & $\mathrm{T}$ & $\mathrm{F}$ & $\begin{array}{l}\text { Fruits is used as } \\
\text { antihelmintic, } \\
\text { gastritis and in } \\
\text { indigestion. }\end{array}$ & $\begin{array}{l}\text { Dhami, 2008; } \\
\text { Acharya and } \\
\text { Acharya, 2009; } \\
\text { Ambu et al., } \\
2020\end{array}$ & 3 \\
\hline 268. & Terminalia chebula Retz. & Harro & Combretaceae & $\mathrm{T}$ & $\mathrm{F}$ & $\begin{array}{l}\text { Fruits are used in } \\
\text { indigestion and } \\
\text { stomach problem. }\end{array}$ & $\begin{array}{l}\text { Dhami, 2008; } \\
\text { Acharya and } \\
\text { Acharya, } 2009\end{array}$ & 2 \\
\hline 269. & Themeda triandra Forssk. & Khar & Poaceae & $\mathrm{H}$ & $\mathrm{L}$ & $\begin{array}{l}\text { Leaf juice is used } \\
\text { to treat gastritis. }\end{array}$ & Acharya, 2012 & 1 \\
\hline 270. & Thymus linearis Benth. & 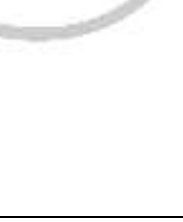 & Lamiaceae & $\mathrm{H}$ & Wp & $\begin{array}{l}\text { Aboveground part } \\
\text { is boiled and given } \\
\text { to treat } \\
\text { stomachache, } \\
\text { gastritis, diarrhoea } \\
\text { and indigestion. }\end{array}$ & $\begin{array}{l}\text { Rokaya et al., } \\
2010\end{array}$ & 1 \\
\hline 271. & $\begin{array}{l}\text { Thysanolaena latifolia (Roxb.ex } \\
\text { Hornem.) Honda }\end{array}$ & & Poaceae & $\mathrm{H}$ & $\mathrm{Up}$ & $\begin{array}{l}\text { Root juice is used } \\
\text { to treat } \\
\text { stomachache and } \\
\text { gastritis }\end{array}$ & Acharya, 2012 & 1 \\
\hline 272. & Tinospora sinensis (Lour.) Merr. & Gurjuko lahara & Menispermaceae & $\mathrm{C}$ & $\mathrm{S}, \mathrm{Up}$ & $\begin{array}{l}\text { Decoction of stem } \\
\text { is used to cure } \\
\text { stomach trouble. } \\
\text { Root extract is } \\
\text { consumed in } \\
\text { gastritis and } \\
\text { diarrhoea. }\end{array}$ & $\begin{array}{l}\text { Joshi et al., } \\
\text { 2011; Malla et } \\
\text { al., 2015; } \\
\text { Singh et al., } \\
\text { 2016; Bhattarai } \\
\text { et al., } 2009\end{array}$ & 4 \\
\hline 273. & Toona ciliata M.Roemer & Tooni & Meliaceae & $\mathrm{T}$ & $\mathrm{B}$ & $\begin{array}{l}\text { Barkmjuice is used } \\
\text { to treat diarrhoea } \\
\text { and dysentery. }\end{array}$ & Dhami, 2008 & 1 \\
\hline 274. & Trichosanthes tricuspidata Lour. & Lahareaanp & Cucurbitaceae & $\mathrm{C}$ & Up & $\begin{array}{l}\text { Root is chewed to } \\
\text { cure constipation. }\end{array}$ & $\begin{array}{l}\text { Limbu \& Rai, } \\
2013\end{array}$ & 1 \\
\hline
\end{tabular}




\begin{tabular}{|c|c|c|c|c|c|c|c|c|}
\hline 275. & Trigonella foenum-graecum $\mathrm{L}$. & Methi & Fabaceae & $\mathrm{H}$ & $\mathrm{F}$ & $\begin{array}{l}\text { Seeds are chewed } \\
\text { raw or grinded } \\
\text { powder is given } \\
\text { with water in } \\
\text { stomachache and } \\
\text { indigestion. }\end{array}$ & $\begin{array}{l}\text { Rokaya et al., } \\
2010\end{array}$ & 1 \\
\hline 276. & Urena lobata L. & Nalukuro & Malvaceae & $\mathrm{H}$ & Wp & $\begin{array}{l}\text { Plant juice is given } \\
\text { to treat stomach } \\
\text { disorders, } \\
\text { dysentery and } \\
\text { diarrhoea. }\end{array}$ & $\begin{array}{l}\text { Manandhar, } \\
\text { 1990, Malla et } \\
\text { al., 2015, } \\
\text { Dhami, 2008 }\end{array}$ & 3 \\
\hline 277. & Urtica dioica $\mathrm{L}$. & Sisnoo & Urticaceae & $\mathrm{S}$ & $\begin{array}{l}\mathrm{L}, \mathrm{S}, \\
\mathrm{Up}\end{array}$ & $\begin{array}{l}\text { Root decoction is } \\
\text { used to cure } \\
\text { stomach disorders. } \\
\text { Cooked leaf or } \\
\text { stem is useful in } \\
\text { gastritis. }\end{array}$ & $\begin{array}{l}\text { Rana et al., } \\
\text { 2015; Rokaya } \\
\text { et al., 2010; } \\
\text { Dhami, } 2008 \\
\end{array}$ & 3 \\
\hline 278. & Valeriana hardwickii Wall. & Nakalijatamansi & Valerianaceae & $\mathrm{H}$ & Up & $\begin{array}{l}\text { Root powder or } \\
\text { paste is given with } \\
\text { hot water in } \\
\text { indigestion, } \\
\text { diarrhoea and } \\
\text { dysentery. }\end{array}$ & $\begin{array}{l}\text { Rokaya et al., } \\
\text { 2010; Ambu et } \\
\text { al., } 2020\end{array}$ & 2 \\
\hline 279. & Valeriana jatamansi Jones. & Sughandawala & Valerianaceae & $\mathrm{H}$ & $\mathrm{L}, \mathrm{Up}$ & $\begin{array}{l}\text { Fresh or dry root } \\
\text { and leaf juice is } \\
\text { used in } \\
\text { stomachache, } \\
\text { indigestion, } \\
\text { diarrhoea and } \\
\text { dysentery. }\end{array}$ & $\begin{array}{l}\text { Rokaya et al., } \\
\text { 2010. Kunwar } \\
\text { \& Adhikari, } \\
\text { 2005. Ambu et } \\
\text { al., } 2020\end{array}$ & 3 \\
\hline 280. & Vitex negundo L. & $=$ & & $\mathrm{S}$ & $\mathrm{L}$ & problem & Dhami, 2008 & 1 \\
\hline 281. & Woodfordia fruticosa (L.) Kurz. & Dhairo & Lythraceae & $\mathrm{S}$ & $\mathrm{Wp}$ & $\begin{array}{l}\text { Flower juice is } \\
\text { given to bloody } \\
\text { dysentery. Leaf is } \\
\text { used to treat } \\
\text { gastritis and } \\
\text { dysentery. Bark } \\
\text { juice and root is } \\
\text { used to treat } \\
\text { stomach problem. }\end{array}$ & $\begin{array}{l}\text { Manandhar, } \\
\text { 1990; } \\
\text { Achharya, } \\
\text { 2012; Dhami, } \\
\text { 2008; Ambu et } \\
\text { al., 2020; } \\
\text { Bhattarai et al., } \\
\text { 2009; Adhikari } \\
\text { et al., 2019 }\end{array}$ & 6 \\
\hline 282. & Wrightia arborea (Dnnst.) mabb. & & Apocynaceae & $\mathrm{T}$ & $\mathrm{F}$ & $\begin{array}{l}\text { Seeds are taken to } \\
\text { treat intestinal pain } \\
\text { having dark stool. }\end{array}$ & $\begin{array}{l}\text { Ambu et al., } \\
2020\end{array}$ & 1 \\
\hline 283. & Youngia japonica (L.) DC. & & Asteraceae & $\mathrm{H}$ & $\mathrm{L}$ & $\begin{array}{l}\text { Leaf juice is taken } \\
\text { in indigestion. }\end{array}$ & Acharya, 2012 & 1 \\
\hline 284. & $\begin{array}{l}\text { Zanthoxylum acanthopodium } \\
\text { DC. }\end{array}$ & Boketimur & Rutaceae & $\mathrm{S}$ & $\mathrm{F}$ & $\begin{array}{l}\text { Fruit is boiled with } \\
\text { water and } \\
\text { consumed during } \\
\text { gastritis, diarrhoea } \\
\text { and dysentery }\end{array}$ & $\begin{array}{l}\text { Singh et al., } \\
2016\end{array}$ & 1 \\
\hline
\end{tabular}




\begin{tabular}{|c|c|c|c|c|c|c|c|c|}
\hline 285. & Zanthoxylum armatum DC. & Timur & Rutaceae & $\mathrm{S}$ & $\mathrm{F}$ & $\begin{array}{l}\text { The fruit is } \\
\text { crushed and taken } \\
\text { with boiled water } \\
\text { to treat abdominal } \\
\text { pain, indigestion, } \\
\text { for apetite and } \\
\text { antihelmintic }\end{array}$ & $\begin{array}{l}\text { Rana et al., } \\
\text { 2015; Joshi et } \\
\text { al., 2011; } \\
\text { Manandhar, } \\
\text { 1990; Singh et } \\
\text { al., 2016; } \\
\text { Shrestha \& } \\
\text { Dhillon, 2003; } \\
\text { Kunwar \& } \\
\text { Adhikari, 2005; } \\
\text { Adhikari et al., } \\
\text { 2019 }\end{array}$ & 7 \\
\hline 286. & Zanthoxylum oxyphyllum Edgew. & Bogyatimur & Rutaceae & $\mathrm{S}$ & $\mathrm{F}$ & $\begin{array}{l}\text { Seed deoction is } \\
\text { used to treat } \\
\text { chronic gastritis. }\end{array}$ & $\begin{array}{l}\text { Shrestha \& } \\
\text { Dhillon, } 2003\end{array}$ & 1 \\
\hline 287. & Zingiber montanum Roxb. & & Zingiberaceae & $\mathrm{H}$ & $\mathrm{Up}$ & $\begin{array}{l}\text { Rhizome is used to } \\
\text { treat gastritis. }\end{array}$ & Acharya, 2012 & 1 \\
\hline 288. & Zingiber officinalae Roscoe & Aduwa & Zingiberaceae & $\mathrm{H}$ & Up & $\begin{array}{l}\text { Rhizome decoction } \\
\text { or rhizome is } \\
\text { boiled and liquid is } \\
\text { taken to treat } \\
\text { stomachache, } \\
\text { constipation and } \\
\text { indigestion. }\end{array}$ & $\begin{array}{l}\text { Rokaya et al., } \\
\text { 2010; Dhami, } \\
\text { 2008; Joshi et } \\
\text { al., 2018; } \\
\text { Bhattarai } \text { et al., } \\
\text { 2009; Adhikari } \\
\text { et al., } 2019\end{array}$ & 6 \\
\hline 289. & Ziziphus mauritiana Lam. & Bayer & Rhamnaceae & $\mathrm{T}$ & $\mathrm{B}$, & $\begin{array}{l}\text { Bark juice is given } \\
\text { in diarrhoea and } \\
\text { dysentery. Fruit is } \\
\text { used to cure } \\
\text { stomachache, } \\
\text { indigestion and } \\
\text { constipation. }\end{array}$ & $\begin{array}{l}\text { Malla } \text { et } \\
\text { al.,2015; } \\
\text { Acharya, 2012; } \\
\text { Dhami, 2008 }\end{array}$ & 3 \\
\hline
\end{tabular}

Note: Habit; $\mathrm{C}=$ Climbers, $\mathrm{H}=$ Herbs, $\mathrm{S}=$ Shrubs, $\mathrm{T}=$ Trees. Used parts; Up= underground parts, Wp= Whole plants, $\mathrm{F}=\mathrm{Fruits}, \mathrm{L}=\mathrm{Leaves}, \mathrm{B}=\mathrm{B}$ ark, $\mathrm{Fl}=$ Flowers, $\mathrm{S}=\mathrm{Stem}, \mathrm{La}=\mathrm{Latex}, \mathrm{Re}=\mathrm{Resin}, \mathrm{Sh}=\mathrm{Shoot}, \mathrm{Sp}=$ Spores, $\mathrm{Bu}=$ Bulbil.

Discussion

Ethnomedicinal plants contain the different bioactive coumpounds that play the key role in treatment of the diseases. The known medicinal uses can thus become the vital source of pharmaceutical drugs for the treatment of many diseases. For developing country like Nepal, health status promotion is almost impossible without developing existing traditional medical systems and incorporating it into the national health care system (Koirala and Khaniya, 2009). Traditional medicinal knowledge is developed from the long process of trial and error and thus this could guide search for drug development
(Karunamoorthi et al., 2012). Among the ethnomedicinal plats used for treatment in gastrointestinal disorders, the species viz. Aconitum heterophyllum, Aconitum ferox, Acorus calamus, Azadirachta indica, Berginia ciliata, Cinnamomum tamala, Curculigo orchioides Dactylorhiza hatagirea, Dioscorea deltoidea, Gaultheria fragrantissima, Juglans regia, Nardostachys grandiflora, Neopicrorhiza scrophulariiflora, Paris polyphylla, Phyllanthus emblica, Piper longum, Rauvolfia serpentine, Rubia manjith, Swertia chirayita, Tinospora sinensis, Valeriana jatamansi and Zanthoxylum armatum are prioritized by Government of 
Nepal for to raise the economic status of nation (DPR 2006; DPR 2016). Among them some are prioritized for agro-technology development.

\section{Conclusion}

This study contributes to the information on ethnomedicinal plant species used in the treatment of gastrointestinal disorders in human beings. In present study, 289 ethnomedicinal plants belonging to 278 angiosperms $(240$ dicots and 38 monocots), five species of gymnosperms and six species of pteridophytes are recorded from Nepal. The plant species, Zanthoxylum armatum is found as the most important ethnomedicinal plant species in the treatment of gastrointestinal disorders as it has highest citation. The indigenous traditional knowledge and practices on the usage of local resources including the ethnomedicinal plants are eroding because of many factors. Bioprospecting of those traditionally used ethnomedicinal plants used by the ethnic communities for long time for treatment of gastrointestinal disorder can be done by conducting the proper research work and to find the novel compounds within them. The ethnomedicinal plants thus need to be conserved and managed in a sustainable way. Moreover, the cultivation technology should be developed for ethnomedicinal plants to avoid the over harvesting of the species in wild.

\section{References}

Acharya, R \& Acharya, K. P. (2009). Ethnobotanical study of medicinal plants used by Tharu community of Parroha vdc, Rupandehi district, Nepal. Scientific World, 7(7): 70-84

2020 November Edition I www.jbino.com I Innovative Association
Acharya, R. (2012). Ethnobotanical study of medicinal plants of Resunga hill used by magar community of Badagaun vdc, Gulmi district, Nepal. Scientific World, 10(10): 54-65.

Adhikari, M., Thapa, R., Kunwar, R. M., Devkota, H. P. \& Poudel, P. (2019). Ethnomedicinal Uses of Plant Resources in the Machhapuchchhre Rural Municipality of Kaski District, Nepal. Medicines, 6(69). doi:10.3390/medicines6020069

Ambu, G., Chaudhary, R. P., Mariotti, M. \& Cornara, L. (2020). Traditional uses of medicinal plants by ethnic people in the Kavrepalanchok District, Central Nepal. Plants, 9(759). doi:10.3390/plants9060759.

Bannerman, R., Burton, J., \& Chen, W. (1983). Traditional Medicine and Health Care Coverage: A Reader for Health Administrators and Practitioners. Geneva: World Health Organization.

Bhattarai, S., Chaudhary, R. P. \& Taylor, R.S. I. (2009). Ethno-medicinal Plants Used by the People of Nawalparasi District, Central Nepal. Our Nature, 7: 82-99.

Dhami, N. (2008). Ethnomedicinal uses of plants in Western Terai of Nepal: A case study of Dekhatbhuli VDC of Kanchanpur District. Medicinal plants in Nepal: An Anthology of Contemporary Research. Pp 164176. Eds . P.K. Jha, S.B. Karmacharya, M. R. Chettri, C.B. Thapa and B.B. Shrestha. Ecological Society, Kathmandu Nepal.

DPR (2006). Plants of Nepal: Fact Sheet. Department of Plant Resources, 
Ministry of Forest and Soil

Conservation,

Thapathali,

Kathmandu, Nepal.

DPR (2016). News Letter (Banaspati Shrota).

Department of Plant Resources, Ministry of Forest and Soil Conservation, Thapathali,

Kathmandu, Nepal. 19 (4).

Ghimire, S. K., Sah, J. P., Shrestha, K. K. \& Bajracharya, D. (1999). Ecological study of some high altitude medicinal and aromatic plants in the Gyasumdo valley, Manang, Nepal. Ecoprint, 6(1): 17-25

Ghimire, K. \& Bastakoti, R. R. (2009).

Ethnomedicinal knowledge and healthcare practices among the Thary of Nawalparasi district in central Nepal. Food and Agriculture Organization of the United Nation. 257: 2066-2072.

Joshi, K., Joshi, R. \& Joshi, A. R. (2011). Indigenous Knowledge and uses of medicinal plants in Machhegaun, Nepal. Indian Journal of Traditional Knowledge, 10(2): 281-286.

Joshi, A., Kalauni, D. \& Bhattarai, S. (2018). Survey on usage of medicinal plants: a case from Chitwan district of Nepal. SAARC J. Agri., 16(2): 129-141. DOI:

https://doi.org/10.3329/sja.v16i2.4026 5

Karunamoorthi, K., Jegajeevanram, K., Vijayalakshmi, J. \& Mengistie, E. (2012. Traditional Medicinal Plants: A Source of Phytotherapeutic Modality in Resource-Constrained Health Care Settings. Journal of Evidence-Based Integrative Medicine. https://doi.org/10.1177/2156587212 460241

Kasper, D. L. \& Harrison, TR. (2005). Harrison's Principles of Internal Medicine. $16^{\text {th }}$ ed. MC

Graw-Hill, Medical pub. Division, Newyork.

Koirala, R. R \& Khaniya, B. N. (2009). Pesent status of: Traditional medicines and medicinal

and aromatic plants related resources and organization in Nepal. Nepal Health Research Council. Ramshahpath Kathmandu, Nepal.

Kunwar, R. M. \& Adhikari, N. (2005). Ethnomedicine of Dolpa district, Nepal: the plants, their

vernacular names and uses. Lyonia 8(1): 43-49.

Limbu, D. K. \& Rai, B. K. (2013). EthnoMedicinal Practices among the Limbu Community in

Limbuwan, Eastern Nepal. Global Journal of Human Social Sciences Multidisciplinary,

13(2).

Malla, B., Gauchan, D. P. \& Chhetri, R. B. (2015). An ethnobotanical study of medicinal plants used by ethnic people in Parbat district of western Nepal. Journal of Ethnopharmacology, (2015), http://dx.doi.org/10.1016/i.jep.2014.1 2.057i.

Manandhar, N. P. (1987). Traditional medicinal plants used by tribals of Lamjung District, Nepal. International Journal of Crude Drug Research, 25(4):

236-240. 
http://dx.doi.org/10.3109/138802087 09055200

Manandhar, N. P. (1990). Medico Botany of Gorkha District, Nepal -An Elucidation of Medicinal Plants. International Journal of Crude Drug Research, 28(1): 17-25.

Paudel, N., Aryal, M. R., Das, B. D., Adhikari, D. C., Rai, P. D. Shrestha, R. (2008). Some medicinal plant from Kathmandu Valley, Central Nepal. International Journal of Scientific Reports, 4(4): 78-81. DOI: http://dx.doi.org/10.18203/issn.24542156.IntJ. SciRep20181390

Rai, S. K. (2004). Medicinal plants used by Meche people of Jhapa District, Eastern Nepal. Our Nature, 200(2):2732.

Rana, S. K., Oli, P. S., \& Rana, H. R. (2015). Traditional botanical knowledge (TBK) on the use of medicinal plants in Sikles area, Nepal. Asian Journal of Plant Science and Research, 5(11): 8-15.

Rawal, R. B. (2004). Marketing Nepal's nontimber forest products: challenges and opportuinities, In local experience based national strategynfor organic production and management of MAPs/ NTFPs in Nepal, eds. N.K. Bhattarai and M. Karki, 87-96. New Delhi: MAPPs/IDRC: Kathmandu GoN, and CCO.
Rokaya, M. B., Munzbergova, Z. \& Timsina, B. (2010). Ethnobotanical study of medicinal plants from the Humla district of western Nepal. Journal of Ethnopharmacology, 130: 485-504.

Shrestha, P. M. \& Dhillon, S. S. (2003). Medicinal plant diversity and use in the highlands of Dolakha district, Nepal. Journal of Ethnopharmacology, 86: 81-96. doi:10.1016/S0378-8741(03)00051-5.

Shrestha, N., Shrestha, S., Koju, L., Shrestha, K. K. \& Wang, Z. (2016). Medicinal plant diversity and traditional healing practices in eastern Nepal. Journal of Ethnopharmacology, 192: 292301.

http://dx.doi.org/10.1016/i.jep.2016.0 7.067.

Singh, A. G., Kumar, A. \& Tewari, D. D. (2012). An ethnobotanical survey of medicinal plants used in Terai forest of western Nepal. Journal of Ethnobiology and Ethnomedicine, 8:19.

Singh, A. G., Kumar, A., Tewari, D. D. \& Bharati, K. A. (2018). New ethnomedicinal claims from Magar community of Palpa district, Nepal. Indian Journal of Traditional Knowledge, 17(3): 499-511.

Ved Prakash. 1998. Indian Medicinal PlantsCurrent Status-I. Ethnobotany, 10: 112-121. 